\title{
Theory of Resonant Raman Scattering in One Dimensional Electronic systems
}

\author{
D.-W. Wang ${ }^{(1)}$, A. J. Millis ${ }^{(2)}$ and S. Das Sarma ${ }^{(3)}$ \\ (1)Physics Department, Harvard University, Cambridge, MA 02138 \\ (2)Department of Physics, Columbia University, New York, NY 10027 \\ (3)Condensed Matter Theory Center, Department of Physics, \\ University of Maryland, College Park, MD 20742
}

(Dated: June 5, 2018)

\begin{abstract}
A theory of resonant Raman scattering spectroscopy of one dimensional electronic systems is developed on the assumptions that (i) the excitations of the one dimensional electronic system are described by the Luttinger Liquid model, (ii) Raman processes involve virtual excitations from a filled valence band to an empty state of the one dimensional electronic system and (iii) excitonic interactions between the valence and conduction bands may be neglected. Closed form analytic expressions are obtained for the Raman scattering cross sections, and are evaluated analytically and numerically for scattering in the polarized channel, revealing a "double-peak" structure with the lower peak involving multispinon excitations with total spin $S=0$ and the higher peak being the conventional plasmon. A key feature of our results is a nontrivial power law dependence, involving the Luttinger Liquid exponents, of the dependence of the Raman cross sections on the difference of the laser frequency from resonance. We find that near resonance the calculated ratio of intensity in the lower energy feature to the intensity in the higher energy feature saturates at a value of the order of unity (times a factor of the ratio of the velocities of the two modes). We explicate the differences between the 'Luttinger liquid' and 'Fermi liquid' calculations of RRS spectra and argue that excitonic effects, neglected in all treatments so far, are essential for explaining the intensity ratios observed in quantum wires. We also discuss other Luttinger liquid features which may be observed in future RRS experiments.
\end{abstract}

\section{INTRODUCTION}

One-dimensional (1D) physics has become increasingly important to condensed matter science in recent decades because many systems, including for example single wall carbon nanotubes ${ }^{1}$, organic conductors ${ }^{2}$, superconducting narowires ${ }^{3}$, spin chains ${ }^{4}$, and even ultracold atoms in highly anisotropic magneto-optical traps ${ }^{5}$, have been developed to levels that allow experimental studies of hitherto unprecedented extent and precision. Among these 1D (or quasi1D) systems, the semiconductor quantum wire structure (QWR) is one of the most important and widely studied, because of its simple band structure and highly tunable doping density ${ }^{6}$. Also, great progress in micro-fabrication techniques has made high quality samples available $\underline{\underline{z}}$.

Theoretically, one dimensional systems are very well understood. The pioneering work of Tomonaga ${ }^{8}$, Luttinger ${ }^{9}$ and Haldane $\frac{10}{}$ along with many other studies has produced an essentially complete understanding of the low energy physics. However, the relation between the theoretical results and experimental data is not as clear and direct as one would like. For example, only a few unambiguous observations of the fundamental concept of spin-charge separation have been reported 11.12 .13 .

Resonant Raman Spectroscopy (RRS) has become a powerful tool for studying the elementary excitations of electrons in many different systems. Applications to low dimensional doped semiconductor nanostructures (twodimensional quantum wells or one-dimensional quantum wires) have been particularly prominent ${ }^{6.14}$. In the usual RRS experiment, schematically represented in Fig. 1(a), external photons are absorbed at one frequency and momentum by exciting an electron from the valence band to the conduction band (step 1) and then emitted at another frequency and momentum via the recombination of the hole in the valence band with another electron in the conduction band (step 2). The final state of the system contains one or more particle-hole pairs excited in the conduction band. The dispersion of these particle-hole pair states may be inferred from the energy and momentum difference between the incident and the scattered photons (Stokes shift), while the nature (collective mode (plasmon) and multipair excitation) may be inferred from the dependence of the scattering amplitude on the polarization of the incoming and outgoing photons and on the energy of the incident photon.

The standard theory of Raman spectroscopy in semiconductors ${ }^{15.16 .17 .18}$, is based on the Fermi liquid (FL) quasiparticle picture and neglects resonance effects. The neglect of resonance effects means that in the unpolarized geometry (identical polarization of incoming and outgoing light) the Raman process couples to the electron density operator so the Raman cross section is proportional to the dynamical structure factor $\frac{18.19}{19}$ of the conduction band electrons. Within Fermi liquid theory and in the long wavelength limit relevant to Raman scattering, the electron structure factor has a delta function peak at the plasmon (CDE) energy and a much weaker feature at a lower energy associated with incoherent particle-hole pairs. The lower energy feature is referred to as the single particle excitation (SPE) 
peak. The calculated RRS intensity therefore has strong spectral peaks when the energy difference between the incident and outgoing photons coincides with the collective CDE mode frequencies at the wavevector defined by the experimental geometry, while the intensity at the SPE energy is much weaker than that at the CDE energy (the ratio is of the order of the square of the ratio of momentum transferred by the light to the Fermi momentum of the electron gas ; for typical experimental relevant parameters, about three orders of magnitude). This theoretical result, however, is in qualitative disagreement with the experimental data ${ }^{20.21 .22}$, in which the polarized spectrum exhibits a "double peak" structure with comparable-intensity peaks at both CDE and SPE modes. This puzzling feature ${ }^{20.21 .22}$ of an ubiquitous strong SPE peak in addition to the expected CDE peak occurs in one, two, and even in three dimensional doped semiconductor nanostructures, for both intrasubband and intersubband excitations. Many theoretical proposals $33,24,25,26,27,28$ have been made to explain this two-peak RRS puzzle. However, two of us have recently $\operatorname{argued}^{29}$ that within the standard theory, which neglects resonance effects, none of the above proposed modified mechanisms theory can even qualitatively explain the experimental data.

But the situation changes when resonance effects are included. In an important paper, Sassetti and Kramer (SK) 24 first proposed that in 1D systems, the prominent lower energy SPE peak is due to "spinon" excitations whose coupling to light is enhanced by resonance effects. The qualitative idea that resonance effects can strongly affect the relative absorption cross-sections of different modes is indeed important. Unfortunately, as we have recently noted, the theory presented in Ref. 24] suffers from two technical flaws. First, it is not self-consistent: it uses a Fermi-liquid-based expression to account for the resonance effects, but a Luttinger-liquid-based expression to account for the conduction band dynamics. As we shall show below (and have already mentioned in a previous brief communication ${ }^{25}$ ) Luttinger liquid physics affects the matrix element in a crucial way. Second, while the SK calculation correctly notes that as resonance is approached the coupling becomes long ranged in space, it omits the equally important fact that the coupling becomes long ranged also in time.

In this paper, which amplifies and extends our previous short communication ${ }^{25}$, we derive and present a complete, closed-form expression for the RRS scattering amplitudes in the Luttinger liquid model and calculate the resulting RRS spectra in different resonance conditions. We treat both the analytically tractable case of short ranged interactions and the physically relevant case of the Coulomb Luttinger liquid. In the Coulomb case we predict an asymmetric broadening of the spectral peak in the higher energy side arising from the curvature of the plasmon dispersion. We find that most aspects of the RRS spectra are similar to those predicted by the Fermi liquid approach. As noted in our previous work $\mathrm{k}^{25}$, characteristic Luttinger effects are revealed in the dependence of the intensities on the difference of the laser frequency from the resonance condition. Going beyond the bosonic expansion developed in our earlier work $^{25}$, we present explicit results for the total spectral weights of the charge boson and spin boson excitations in the polarized RRS channel. Far from resonance we find that the spin-singlet mode at energy, $\omega=q v_{F}$, has spectral weight much smaller than that of the charge boson (i.e. plasmon); however as resonance is approached the weights in the two contributions become comparable. We explain the difference between the Luttinger liquid results presented here and the 'Fermi Liquid' results presented previously 15.16.17.18.23.30. Our results remain inconsistent with present experimental data, so we argue that Luttinger liquid effects have not yet been unambiguously detected in RRS experiments. One possibility is that excitonic effects, neglected in the present and previous treatments, are important.

The paper is organized as follows: In Sec. Пwe develop a general theory of Resonant Raman Scattering in Luttinger liquids. We then apply our theory to calculate the spectrum for short- and long-ranged electron-electron interaction by using bosonic expansion method in Sec. III] In Sec. IV] we go beyond the bosonic expansion and calculate the full spectral weights of the charge boson and spin-singlet excitations in the polarized channel. We compare our calculation to the previous 'Fermi liquid' calculations in Sec. $\nabla$ We critically discuss the assumptions and resonance effects of our results and compare them with the present experimental data in Sec. VI Finally we summarize our results in Sec. VII]

\section{RESONANT RAMAN SCATTERING CROSS SECTION OF A LUTTINGER LIQUID}

In this section we present a derivation of the RRS scattering cross section for a Luttinger liquid. We consider an idealized model of a quasi-one-dimensional system, in which the electron motion in the transverse directions ( $y$ and $z$ ) is assumed to be completely frozen due to a strong confinement potential in both conduction and valence band(in other words, we consider only the lowest conduction and highest valence subband). Our results are likely to apply also to the case where the resonance occurs via some other intermediate state, but we have not considered this case explicitly.

The longitudinal ( $x$ direction) motion is assumed to be free without defects or disorder. An important feature of quantum wires is the Coulomb interaction, which is typically unscreened and leads to a long ranged interaction with a well-known characteristic form ${ }^{25}$ involving the transverse wavefunction ${ }^{30}$. The scale dependence of the unscreened 
Coulomb interaction complicates considerably the analysis of Luttinger liquid formulae, but as will be seen also leads to the appearance of additional structures in the predicted RRS spectra. In this section we derive an expression for the RRS cross section of a general model with arbitrary electron interaction. Subsequent sections present results for short ranged interactions (where the analysis can be carried through in considerable analytical detail) and the more physically relevant long ranged interaction, which requires additional approximations.

The appropriate theoretical starting point for analysis of Raman scattering is the following general Hamiltonian

$$
H_{\mathrm{tot}}=H_{V}+H_{L L}+H_{c v}+H_{\mathrm{int}}^{k, \omega},
$$

where $H_{V}$ and $H_{L L}$ are the Hamiltonians for the valence and conduction bands respectively, and $H_{\text {int }}^{k, \omega}$ gives the coupling of these carriers to externally applied radiation. $H_{c v}$ describes the excitonic interaction between conduction band electrons and valence band holes, and may be important in certain conditions $\frac{31}{1}$. We will assume excitonic effects are irrelevant in the energy regime of interest and will therefore set $H_{c v}=0$ throughout this paper. In this case, the RRS process involves only one electron excited out of the valence band, so that interactions in the valence band can be also neglected. We consider a single one-dimensional conduction sub-band and a valence subband, linearize the dispersion about the Fermi level and include an (at this stage arbitrary) interaction between conduction band electrons. These considerations imply

$$
\begin{aligned}
H_{V} & =\sum_{r, p, s} E_{r, p}^{V} v_{r, p, s}^{\dagger} v_{r, p, s} \\
H_{L L} & =\sum_{r, p, s} v_{F}\left(r p-k_{F}\right) c_{r, p, s}^{\dagger} c_{r, p, s}+\frac{1}{2 L} \sum_{r_{1}, r_{2}, s_{1}, s_{2}} \sum_{q, p_{1}, p_{2}} V(q) c_{r_{1}, p_{1}-q / 2, s_{1}}^{\dagger} c_{r_{2}, p_{2}+q / 2, s_{2}}^{\dagger} c_{r_{2}, p_{2}-q / 2, s_{2}} c_{r_{1}, p_{1}+q / 2, s_{1}},
\end{aligned}
$$

where the conduction band Hamiltonian can be bosonized as in the standard Luttinger liquid theory ${ }^{32}$, yielding

$$
H_{L L}=\sum_{p}\left(\omega_{p}^{\rho} b_{p}^{\dagger} b_{p}+\omega_{p}^{\sigma} \sigma_{p}^{\dagger} \sigma_{p}\right)
$$

We approximate the valence band energy, $E_{r, p}^{V}$, by a linear dispersion about the Fermi wavevector of electrons in the conduction band:

$$
E_{r, p}^{V} \approx-\Omega_{r r s}-v_{F}^{V}\left(r p-k_{F}\right)
$$

where $\Omega_{r r s} \equiv E_{F}^{c}+E_{F}^{V}+E_{g}$ is the RRS resonance energy (see Fig. 廿(a)). Note that in this expression it is assumed that the valence band is also one dimensional. If transverse motion in the valence band is important, these degrees of freedom should be integrated out, which will broaden the valence band propagator. (Note that the structure of the quantum wire system means that momentum transverse to the wire need not be conserved in an optical transition). $c_{r, p, s}^{\dagger}$ and $v_{r, p, s}^{\dagger}$ are creation operators of electrons of chirality $r= \pm 1$ (left/right moving), wavevector $p$ and spin $s$, in conduction band and valence band respectively; $b_{p}^{\dagger}$ and $\sigma_{p}^{\dagger}$ are charge boson and spin boson creation operators in Luttinger liquid theory (see Refs. [10,32] for a general review). $v_{F}\left(k_{F}\right)$ is the Fermi velocity(wavevector) of conduction band electrons. $V(q)$ is the effective 1D electron-electron interaction within the conduction band. In Eq. (3), the charge (spin) boson energy, $\omega_{p}^{\rho}\left(\omega_{p}^{\sigma}\right)$, is related to the interaction $V(q)$ via ${ }^{32}$

$$
\begin{aligned}
\omega_{p}^{\rho} & =|p| v_{F} \sqrt{1+\frac{V(p)}{\pi v_{F}}}, \\
\omega_{p}^{\sigma} & =|p| v_{F},
\end{aligned}
$$

where we have assumed that electron-electron interaction is spin-independent so that the spin boson velocity is the same as noninteracting electron Fermi velocity.

Finally, for the electron-photon interaction Hamiltonian, $H_{\text {int. }}$, we consider only the three-leg vertex for which photon number is not conserved (see Fig. 1(b) and Refs. 30 33 34]), and neglect the four-leg vertex where photon number is conserved (see Fig. प(c)), because the contribution of the latter does not give rise to resonance effects and is thus ${ }^{30}$ much smaller than the contribution of the three-leg vertex in near resonance conditions. We also represent the radiation by a classical field, so that

$$
\begin{aligned}
H_{\mathrm{int}}^{k, \omega} & =\sum_{r, p, s, s^{\prime}}\left(g_{1} \delta_{s, s^{\prime}}+g_{2} \delta_{s,-s^{\prime}}\right)\left\{\left[c_{r, p, s}^{\dagger}(t) v_{r, p-k, s^{\prime}}(t)+v_{r, p, s^{\prime}}^{\dagger}(t) c_{r, p-k, s}(t)\right] e^{-i \omega t}\right. \\
& \left.+\left[c_{r, p, s}^{\dagger}(t) v_{r, p+k, s^{\prime}}(t)+v_{r, p, s^{\prime}}^{\dagger}(t) c_{r, p+k, s}(t)\right] e^{i \omega t}\right\}
\end{aligned}
$$


where $\omega$ and $k$ are energy and wavevector of the photon interacting with electrons. $g_{1}$ and $g_{2}$ are coupling constants for non-spin-flip and spin-flip scattering respectively, and their actual values are not important in our study. In the remainder of this section, we present an explicit derivation of expressions for the non-spin-flip (polarized spectrum) RRS cross section following from Eqs. (2), (3) and (6).

Following our earlier work (Ref. 25 30]), we use second order time-dependent perturbation theory to calculate the rate at which $H_{\text {int }}^{k, \omega}$ causes transitions from the ground state $|0\rangle$ to some state $|n\rangle$ in which the valence band is filled and the conduction band state has changed. One finds

$$
W(q, \nu ; \Omega)=\lim _{T \rightarrow \infty} \frac{1}{T} \sum_{n}\left|\int_{-T / 2}^{T / 2} d t_{1} \int_{-T / 2}^{t_{1}} d t_{2}\left\langle n\left|H_{\mathrm{int}}^{q / 2, \Omega+\nu / 2}\left(t_{1}\right) H_{\mathrm{int}}^{-q / 2, \Omega-\nu / 2}\left(t_{2}\right)\right| 0\right\rangle\right|^{2},
$$

where $q$ and $\nu$ are photon wavevector and frequency shifts after Raman scattering and $\Omega$ is the mean frequency of incident and scattered photons during the process. We choose the backward scattering channel so that all wavevectors involved are along the wire direction for simplicity. Because the valence band is filled in the ground state, the part of the correlator involving valence electrons is simple:

$$
\begin{aligned}
& \left\langle v_{r_{1}, p_{1}-q / 2, s_{1}}^{\dagger}\left(t_{2}^{\prime}\right) v_{r_{2}, p_{2}, s_{2}}\left(t_{1}^{\prime}\right) v_{r_{3}, p_{3}, s_{3}}^{\dagger}\left(t_{1}\right) v_{r_{4}, p_{4}-q / 2, s_{4}}\left(t_{2}\right)\right\rangle_{0} \\
& =\delta_{r_{1}, r_{2}} \delta_{r_{3}, r_{4}} \delta_{p_{1}, p_{2}+q / 2} \delta_{p_{3}, p_{4}-q / 2} \delta_{s_{1}, s_{2}} \delta_{s_{3}, s_{4}} e^{i E_{r_{1}, p_{1}}^{V}\left(t_{2}^{\prime}-t_{1}^{\prime}\right)} e^{i E_{r_{3}, p_{3}}^{V}\left(t_{1}-t_{2}\right)} .
\end{aligned}
$$

Using the space-time translational symmetry, Eq. (7) can be further simplified by representing fermion operators in coordinate space:

$$
W(q, \nu ; \Omega)=\lim _{L \rightarrow \infty} \int_{0}^{L} d R \int_{-\infty}^{\infty} d T e^{i(\nu T-q R)}\left\langle\widehat{O}^{\dagger}(R, T) \widehat{O}(0,0)\right\rangle_{0}
$$

Here $\langle\cdots\rangle_{0}$ are the expectation values on the ground state wavefunction and

$$
\widehat{O}(R, T)=\sum_{r, s} \int_{0}^{L} d x \int_{0}^{\infty} d t \phi(x, t) \psi_{r, s}(R+x / 2, T+t / 2) \psi_{r, s}^{\dagger}(R-x / 2, T-t / 2),
$$

and

$$
\phi(x, t)=\frac{e^{i \Omega t}}{L} \sum_{p} e^{i\left(E_{p}^{V} t-p x\right)}=e^{i\left(\Omega-\Omega_{r r s}+v_{F}^{V} k_{F}\right) t} \delta\left(x+r v_{F}^{V} t\right) .
$$

Eqs (91)-(11) are our fundamental results. They show that the RRS process creates an electron-hole pair separated in space by $x$ and in time by $t$, with the amplitude for a given space-time separation controlled by the function $\phi(x, t)$, which is essentially the propagator for the valence-ban hole. Far from resonance $\left(\left|\Omega-\Omega_{r r s}\right| \gg v_{F}^{V} k_{F}\right), \phi(x, t)$ is short ranged in both $x$ and $t$, so that $\widehat{O}$ becomes similar to the ordinary density operator and $W$ becomes a densitydensity correlation function ${ }^{29.30}$. As the mean photon energy is tuned closer to the resonance condition, $\phi$ becomes longer ranged, so that $\widehat{O}$ becomes nonlocal in both space and time. This non-locality will be seen to give rise to the interesting resonance effects, by allowing the light to couple to something other than the dynamical structure factor. We also observe that up to this point we have not made use of the one dimensionality in any important way: the equations may easily be generalized to two and three dimensions. Previous theories $\frac{15,16,17.18,23,24,25,26,28,29.33}{\text { of the }}$ RRS process have been based on similar expressions but with a function $\phi$ which essentially forces $t=0$ (i.e. no retardation effects). We shall see below that the time dependence is very important.

We now incorporate the special features of one-dimensional physics by using the standard ${ }^{32}$ bosonization representation of electron operators in Eq. (10)

$$
\psi_{r, s}(x, t)=\frac{e^{i r k_{F} x}}{\sqrt{2 \pi \alpha}} \exp \left(i \sum_{p>0} \sqrt{\frac{\pi}{p L}}\left\{e^{-p \alpha / 2+i r p x}\left[\cosh \theta_{p} b_{r p}(t)-\sinh \theta_{p} b_{-r p}^{\dagger}(t)+s \sigma_{r p}(t)\right]+\text { h.c. }\right\}\right),
$$

where $\exp \left(-2 \theta_{p}\right)=\omega_{p}^{\rho} / p v_{F}=\sqrt{1+V(p) / \pi v_{F}}$ is the momentum dependent LL exponent, and $\alpha \rightarrow 0^{+}$is a convergence factor. Substituting Eq. (III) into Eq. (10) and using the following identity for linear boson operators, $A$ and $B$ (valid when $[A, B]$ commutes with both $A$ and $B$, the colons denote normal ordering and the $<>_{0}$ denotes expectation value with respect to the ground state of $H_{L L}$ ):

$$
e^{A} e^{B}=\left\langle e^{A} e^{B}\right\rangle_{0}: e^{A+B}:,
$$


we can rewrite $\widetilde{O}$ as

$$
\widehat{O}(R, T)=L \sum_{r, s} \int_{0}^{\infty} d t e^{i\left(\Omega-\Omega_{r r s}\right) t} G_{r, s}\left(-r v_{F}^{V} t, t\right): e^{\Phi_{r s, \rho}\left(R,-r v_{F}^{V} t ; T, t\right)}:: e^{\Phi_{r s, \sigma}\left(R,-r v_{F}^{V} t ; T, t\right)}:,
$$

where we have separately normal ordered the charge and spin boson operators and $G_{r \sigma}(x, t)=$ $\lim _{\alpha \rightarrow 0}(2 \pi \alpha)^{-1}\left\langle\psi_{r \sigma}(x, t) \psi_{r \sigma}^{\dagger}(0,0)\right\rangle$ is the electron Green's function in the conduction band. The phase operators, $\Phi_{\rho}$ and $\Phi_{\sigma}$ are respectively

$$
\begin{aligned}
\Phi_{r s, \rho}(R, x ; T, t) & =2 \sum_{p>0} e^{-\alpha p / 2} \sqrt{\frac{\pi}{p L}}\left\{-\sinh \theta_{p} \sin \left[p\left(r x+v_{p}^{\rho} t\right) / 2\right]\left[b_{-r p}^{\dagger} e^{i p\left(r R+v_{p}^{\rho} T\right)}+\text { h.c. }\right]\right. \\
& \left.+\cosh \theta_{p} \sin \left[p\left(r x-v_{p}^{\rho} t\right) / 2\right]\left[b_{r p}^{\dagger} e^{-i p\left(r R-v_{p}^{\rho} T\right)}+\text { h.c. }\right]\right\} \\
\Phi_{r s, \sigma}(R, x ; T, t) & =2 s \sum_{p>0} e^{-\alpha p / 2} \sqrt{\frac{\pi}{p L}}\left\{\sin \left[p\left(r x-v_{F}^{V} t\right) / 2\right]\left[\sigma_{r p}^{\dagger} e^{-i p\left(r R-v_{F}^{V} T\right)}+\text { h.c. }\right]\right\} .
\end{aligned}
$$

To calculate the expectation value of a product of normal orderings, we use two additional identities for the linear bosonic operators (valid under the same condition as Eq. (12))

$$
\left\langle e^{A} e^{B}\right\rangle_{0}=\left\langle e^{A+B}\right\rangle_{0} e^{[A, B] / 2}
$$

and

$$
\left\langle e^{A}\right\rangle_{0}=e^{\left\langle A^{2}\right\rangle_{0} / 2}
$$

so that

$$
\begin{aligned}
\left\langle: e^{A}:: e^{B}:\right\rangle_{0} & =e^{-\left\langle A^{2}\right\rangle_{0} / 2} e^{-\left\langle B^{2}\right\rangle_{0} / 2}\left\langle e^{A} e^{B}\right\rangle_{0} \\
& =e^{-\left\langle A^{2}\right\rangle_{0} / 2} e^{-\left\langle B^{2}\right\rangle_{0} / 2}\left\langle e^{A+B}\right\rangle_{0} e^{[A, B] / 2} \\
& =e^{\langle A B\rangle_{0}} .
\end{aligned}
$$

Combining all the results from Eq. (9) to Eq. (17) and defining the average photon frequency relative to the resonance frequency $\Omega_{r r s}$ as $\tilde{\Omega}=\Omega-\Omega_{r r s}$, we obtain

$$
\begin{aligned}
W(q, \nu ; \tilde{\Omega}) & =\sum_{r_{1}, r_{2}} \sum_{s_{1}, s_{2}} W_{s_{1}, s_{2}}^{r_{1}, r_{2}}(q, \nu ; \tilde{\Omega}) \\
W_{s_{1}, s_{2}}^{r_{1}, r_{2}}(q, \nu ; \Omega) & =\int_{-\infty}^{\infty} d T \int d R e^{i \nu T-i q R} \int_{0}^{\infty} d t_{1} f_{r_{1} s_{1}}^{*}\left(t_{1} ; \tilde{\Omega}\right) \int_{0}^{\infty} d t_{2} f_{r_{2} s_{2}}\left(t_{2} ; \tilde{\Omega}\right) \\
& \times\left\{\exp \left[\left\langle\Phi_{r_{1} s_{1}, \rho}\left(R,-r_{1} v_{F}^{V} t_{1} ; T, t_{1}\right) \Phi_{r_{2} s_{2}, \rho}\left(0,-r_{2} v_{F}^{V} t_{2} ; 0, t_{2}\right)\right\rangle_{0}\right]\right. \\
& \left.\times \exp \left[\left\langle\Phi_{r_{1} s_{1}, \sigma}\left(R,-r_{1} v_{F}^{V} t_{1} ; T, t_{1}\right) \Phi_{r_{2} s_{2}, \sigma}\left(0,-r_{2} v_{F}^{V} t_{2} ; 0, t_{2}\right)\right\rangle_{0}\right]-1\right\},
\end{aligned}
$$

where

$$
f_{r s}(t ; \tilde{\Omega})=e^{i \tilde{\Omega} t} G_{r s}\left(-r v_{F}^{V} t, t\right) .
$$

The -1 in the last line of Eq. (18) arises from normal ordering ${ }^{10}$.

The charge and spin part of the expectation values in Eq. (18) can be calculated respectively to be (at zero temperature):

$$
\begin{aligned}
& \left\langle\Phi_{r_{1} s_{1}, \rho}\left(R, x_{1}, T, t_{1}\right) \Phi_{r_{2} s_{2}, \rho}\left(0, x_{2}, 0, t_{2}\right)\right\rangle_{0} \\
& =2 r_{1} r_{2} \int_{0}^{\infty} \frac{d p}{p} e^{-\alpha p / 2} e^{-i p v_{p} T}\left\{\sin \left[p\left(x_{1}-r_{1} v_{p}^{\rho} t_{1}\right) / 2\right] \sin \left[p\left(x_{2}-r_{2} v_{p}^{\rho} t_{2}\right) / 2\right] C_{r_{1}, p} C_{r_{2}, p} e^{i p R}\right. \\
& \left.+\sin \left[p\left(x_{1}+r_{1} v_{p}^{\rho} t_{1}\right) / 2\right] \sin \left[p\left(x_{2}+r_{2} v_{p}^{\rho} t_{2}\right) / 2\right] C_{-r_{1}, p} C_{-r_{2}, p} e^{-i p R}\right\},
\end{aligned}
$$

where $C_{r, p} \equiv \cosh \theta_{p}\left(\sinh \theta_{p}\right)$ for $r=+1(-1)$, and

$$
\begin{aligned}
& \left\langle\Phi_{r_{1} s_{1}, \sigma}\left(R, x_{1}, T, t_{1}\right) \Phi_{r_{2} s_{2}, \sigma}\left(0, x_{2}, 0, t_{2}\right)\right\rangle_{0} \\
& =2 s_{1} s_{2} \delta_{r_{1}, r_{2}} \int_{0}^{\infty} \frac{d p}{p} e^{-\alpha p / 2} \sin \left[p\left(x_{1}-v_{F} t_{1}\right) / 2\right] \sin \left[p\left(x_{2}-v_{F} t_{2}\right) / 2\right] e^{i r_{1} p R-i p v_{F}^{V} T}
\end{aligned}
$$

Eqs. (18)-(21) represent a complete solution to the RRS cross section of a Luttinger liquid in the absence of excitonic effects. We will evaluate them analytically and numerically in the following sections. 


\section{LEADING ORDER EFFECTS IN BOSONIC EXPANSION}

\section{A. Overview}

Although Eq. (18) is a complete closed-form expression for the RRS cross section, it requires a numerical evaluation which is not simple because final results are obtained by cancellation of rapidly oscillating terms. It is instructive to consider the analytical approximation obtained by expanding the exponential factors in Eq. (18) in a Taylor series in $\langle\Phi \Phi\rangle^{n}$ ( $n$ is an integer) and then evaluating the series term-by-term. Note that in the present problem (unlike in the evaluation of the electron Green function) each term in the expansion is finite because the sine function in Eqs. (20) and (21) removes the $1 / p$ divergence in small $p$ region and, by oscillating, ensures the convergence at large $p$. The expansion approach has a simple physical interpretation: the term of order $n$ in the expansion corresponds to a final state with $n$ excited bosons.

Subsection $B$ shows results obtained on the assumption of short-ranged interaction between electrons in conduction band, i.e. $V(p)=V_{0}$ with an appropriate momentum cut-off. The well-known analytical methods of LL theory can then be applied, yielding a physical understanding of the features of RRS spectrum. Subsection $C$ presents results obtained for the unscreened Coulomb interaction.

\section{B. Results for short-ranged interaction}

For simplicity here we assume that the valence band electron velocity at the Fermi momentum of the conduction band, $v_{F}^{V}$, is much less than the conduction band velocity, $v_{F}$, and can be neglected. In this approximation (which does not affect any essential results),

$$
f(t, \bar{\Omega})=e^{i \tilde{\Omega} t} G_{r s}(x=0, t) \sim \frac{e^{i \tilde{\Omega} t}}{2 \pi i \sqrt{v_{\rho} v_{F}}} \frac{1}{t\left(i E_{0} t\right)^{\alpha_{L L}}},
$$

where

$$
\alpha=\frac{1}{4}\left(\frac{v_{\rho}}{v_{F}}+\frac{v_{F}}{v_{\rho}}-2\right)=\sinh ^{2} \theta_{\rho}
$$

is the Luttinger exponent and $E_{0}$ is an energy cut-off above which the Luttinger liquid model ceases to describe the physics. (Note that here we follow the standard convention in the literature ${ }^{32}$ and this $\alpha$ is nothing to do with the convergent factor used in Eq. (III).) $E_{0}$ is expected to be roughly of the order of the conduction band Fermi energy, $E_{F}^{c 32}$. The time integrals can now be reduced to gamma functions, and the expansion evaluated order by order.

We first show the results for the one-boson contribution in the non-spin-flip process. Here the spin boson term is canceled by the spin sum as required for the conservation of angular momentum. We have

$$
W_{1}(q, \nu ; \tilde{\Omega})=\frac{\Gamma^{2}(-\alpha)}{2 q v_{F} v_{\rho}}\left|A_{1}(\tilde{\Omega}, q, \alpha)\right|^{2} e^{2 \theta_{\rho}} \delta\left(\nu-q v_{\rho}\right),
$$

where the function $A_{1}$ is

$$
A_{1}(\tilde{\Omega}, p, \alpha)=\left(\frac{\tilde{\Omega}-q v_{\rho} / 2}{E_{0}}\right)^{\alpha}-\left(\frac{\tilde{\Omega}+q v_{\rho} / 2}{E_{0}}\right)^{\alpha} .
$$

Thus the one boson term is a delta function at the plasmon energy, i.e. it gives the conventional CDE contribution to the scattering cross section. The amplitude for excitation depends on the difference of the mean photon frequency from the resonance condition and on the transferred momentum $q$. Far from resonance, $\omega_{q}=q v_{\rho} \ll|\tilde{\Omega}|$ and $W_{1} \propto|\tilde{\Omega}|^{2 \alpha-2}$, while at resonance $\tilde{\Omega}=0$ and $W_{1} \propto \sin ^{2}(\pi \alpha / 2)$. Thus LL effects enter the CDE part $\left(\nu \sim q v_{\rho}\right)$ of the spectrum in two ways (for short-ranged interaction): first, far from resonance, they changes the frequency dependence of spectral weights from $\tilde{\Omega}^{-2}$, the noninteracting result, to $\tilde{\Omega}^{-2+2 \alpha}$. Second, near resonance $\left(\tilde{\Omega}<v_{\rho} q / 2\right)$ Luttinger Liquid effects resolve the singularities which are found in the standard Fermi-liquid expressions for the matrix elements: see Sec. $\nabla$ for a more detailed discussion. 
For the second order (i.e. two-boson) contribution, we obtain

$$
\begin{aligned}
W_{2}(q, \nu ; \tilde{\Omega}) & =\frac{\Gamma^{2}(-\alpha)}{v_{F}^{2}}\left\{\int_{0}^{q} \frac{d p}{p(q-p)}\left|A_{2}^{\rho}(\tilde{\Omega}, p, q-p, \alpha)\right|^{2} \cosh ^{2}\left(2 \theta_{\rho}\right) \delta\left(\nu-q v_{\rho}\right)\right. \\
& +2 \int_{q}^{\infty} \frac{d p}{p(p-q)}\left|A_{2}^{\rho}(\tilde{\Omega}, p, p-q, \alpha)\right|^{2} \sinh ^{2}\left(2 \theta_{\rho}\right) \delta\left(\nu-p v_{\rho}-(p-q) v_{\rho}\right) \\
& \left.+\int_{0}^{q} \frac{d p}{p(q-p)}\left|A_{2}^{\sigma}(\tilde{\Omega}, p, q-p, \alpha)\right|^{2} \delta\left(\nu-q v_{F}^{\sigma}\right)\right\}
\end{aligned}
$$

with $(\alpha=\rho, \sigma)$

$$
\begin{aligned}
A_{2}^{\alpha}\left(\tilde{\Omega}, p_{1}, p_{2}, \alpha\right) & =\left(\frac{\tilde{\Omega}-p_{1} v_{\alpha} / 2-p_{2} v_{\alpha} / 2}{E_{0}}\right)^{\alpha}-\left(\frac{\tilde{\Omega}+p_{1} v_{\alpha} / 2-p_{2} v_{\alpha} / 2}{E_{0}}\right)^{\alpha} \\
& -\left(\frac{\tilde{\Omega}-p_{1} v_{\alpha} / 2+p_{2} v_{\alpha} / 2}{E_{0}}\right)^{\alpha}+\left(\frac{\tilde{\Omega}+p_{1} v_{\alpha} / 2+p_{2} v_{\alpha} / 2}{E_{0}}\right)^{\alpha} .
\end{aligned}
$$

Note that for large $\tilde{\Omega}$ (far from resonance) $W_{2} \sim \tilde{\Omega}^{-4+2 \alpha}$ so that in this limit the two boson term is small compared to the one-boson term, confirming the validity of the expansion in the far-from resonance region. The first term in Eq. (26) gives a renormalization of the strength of the 'CDE' pole. The second and third terms produce new affects appearing at second order: first near the CDE peak, branch mixing (second line of Eq. (26)) processes appear, leading to a continuum absorption beginning at the CDE threshold, $\nu=q v_{\rho}$. Secondly the spin singlet combination of spin bosons (third line) can be excited via the two spinon, $\langle\sigma \sigma, \sigma \sigma\rangle$ process, giving rise to the so-called "SPE" mode at $\nu=q v_{F}$.

In Fig. 2 we show the perturbatively calculated LL polarized RRS spectra including one and two boson contributions to the Raman intensity, for a particular choice of Luttineger exponent The data are plotted as a function of energy transferred at fixed momentum transfer $q$; the different graphs show results for different values of the laser frequency relative to the resonance condition. One observes that (i) the overall spectral weight decays very fast as $\Omega$ is moved off resonance, and (ii) the "SPE" peak is only noticeable near resonance. (iii) The continuum structure arising from the second term in Eq. (26) is visible but not very distinct. We also remark that the three boson term in the expansion (not calculated in paper) will produce an additional continuum structure lying between the "SPE" $\left(\omega=q v_{F}\right)$ and the plasmon $\left(\omega=q v_{\rho}\right)$ energies, due to the coupling between charge and spin bosons via the RRS process.

\section{Long-ranged Coulomb interaction}

We now consider the modifications arising in the physically relevant case of a long range, unscreened Coulomb interaction. As discussed elsewhere ${ }^{37}$ this corresponds to a scale dependent Luttinger interaction parameter, $\alpha_{q}=$ $\alpha_{0} \ln ^{1 / 2}\left(q_{0} / q\right)$ (for $\left.q \ll q_{0}\right)$ and the charge mode plasmon energy $y^{37}$,

$$
\omega_{q}^{\rho} \sim 4 \alpha_{0} v_{F} q \ln ^{1 / 2}\left(q_{0} / q\right)
$$

Here $q_{0} \sim 2.5 / a$ ( $a$ is the typical width of QWR) is the momentum scale below which Coulomb effects become important and $\alpha_{0}=\sqrt{e^{2} / 2 \epsilon_{0} v_{F} \pi^{37}}$ for typical QWR structure. In the GaAs QWR material which was recently studied $^{35}$, typical LL parameters are $\alpha_{0} \sim 0.389$ and $q_{0} \sim 1.4 \times 10^{6} \mathrm{~cm}^{-1}$.

The scale dependence of the interaction prevents analytic evaluation of the Luttinger liquid formulae, but the bosonic expansion can still be carried out, although both the integrals defining $f(t, \tilde{\Omega})$ and those defining the $\Phi$ must be evaluated numerically.

In Fig. [3] we show the one and two boson contributions to the polarized RRS intensity of 1D QWR using Coulomb interaction parameters corresponding to the system studied in Ref ${ }^{35}$. The basic features of the results are similar to the short-ranged interaction, having one weak singlet spinon peak at the "SPE" $\operatorname{spinon}$ energy $\omega=q v_{\sigma}=q v_{F}$ arising from the two coupled spin bosons, and one strong charge boson peak at the plasmon energy $\omega=q v_{q}$. Their relative strength varies according to the resonance condition. Off resonance, the two-boson-singlet-spinon peak is much weaker than the charge boson plasmon peak, while their total spectral weight is also very small compared with the near resonance result. 
Near resonance, an additional feature appears: the two-charge-boson contribution gives another resonance peak at $\omega=2 \omega_{q / 2}^{\rho}>\omega_{q}^{\rho}$, arising from the nonlinear $q$ dependence of $\omega_{q}^{\rho}$ due to the long-ranged Coulomb interaction. The resulting curvature acts to broaden the spectral weight on the higher energy side. Next order bosonic terms will contribute the spin-charge mixing weights from $\langle\sigma \sigma \rho, \rho \sigma \sigma\rangle$ type higher order correlations. This will introduce a continuum of relatively weak spectral weight which is located in the energy region between the spinon and the plasmon energies, as noted previously.

\section{BEYOND THE BOSONIC EXPANSION}

We now consider effects beyon the bosonic expansion in the analytically tractable finite range interaction model. We begin with the exact expression, Eqs. (20)-(21). The values of mean position $R$ and time $T$ are expected to be roughly inversely proportional to the momentum $q$ and frequency $\nu$ transferred to the system. If $\nu$ and $q$ are small compared to the basic scales $\left(E_{F}\right.$ and $\left.k_{F}\right)$ we may use a long wavelength approximation to evaluate the phase factors in the standard way, obtaining

$$
\begin{aligned}
W_{s_{1}, s_{2}}^{r_{1}, r_{2}}(q, \nu ; \tilde{\Omega}) & =\int_{0}^{\infty} d t_{1} f_{r_{1} s_{1}}^{*}\left(t_{1} ; \tilde{\Omega}\right) \int_{0}^{\infty} d t_{2} f_{r_{2} s_{2}}\left(t_{2} ; \tilde{\Omega}\right) \int_{-\infty}^{\infty} d T e^{i \nu T} \int_{-\infty}^{\infty} d R e^{-i q R} \\
& \times\left\{\left[F_{r_{1}, r_{1}}^{\sigma}\left(r_{1} R-v_{\sigma} T ; t_{1}, t_{2}\right)\right]^{\alpha_{\sigma}}\left[F_{r_{1}, r_{2}}^{\rho}\left(R-v_{\rho} T ; t_{1}, t_{2}\right)\right]^{\alpha_{\rho}^{(1)}}\right. \\
& \left.\times\left[F_{r_{1}, r_{2}}^{\rho}\left(-\left(R+v_{\rho} T\right) ; t_{1}, t_{2}\right)\right]^{\alpha_{\rho}^{(2)}}-1\right\}
\end{aligned}
$$

where the exponents are, $\alpha_{\sigma}=\frac{1}{2} \delta_{r_{1}, r_{2}} s_{1} s_{2}, \alpha_{\rho}^{(1)}=\frac{1}{2} C_{r_{1}} C_{r_{2}}$, and $\alpha_{\rho}^{(2)}=\frac{1}{2} C_{-r_{1}} C_{-r_{2}}$. The $F$ function is:

$$
F_{r_{1}, r_{2}}^{a}\left(X ; t_{1}, t_{2}\right)=\frac{\left(v_{r_{1}}^{a} t_{1}+v_{r_{2}}^{a} t_{2}\right)^{2}-(X+i \alpha)^{2}}{\left(v_{r_{1}}^{a} t_{1}-v_{r_{2}}^{a} t_{2}\right)^{2}-(X+i \alpha)^{2}}
$$

where $v_{r}^{a}=\left(v_{a}+r v_{F}^{V}\right) / 2$ for $a=\sigma, \rho\left(v_{\sigma}=v_{F}\right)$.

We have not attempted a direct evaluation of this expression. Instead, we note that the spectrum is expected on general grounds to consist of two delta functions, at the CDE and SPE energies, and two continua. The delta functions are the features most easily observable experimentally, and it is possible to obtain convenient expressions for their weights. We note that terms giving rise to delta functions at the CDE energy or SPE energy must be functions only of $R-v_{\rho} T$ and $R-v_{\sigma} T$ respectively. To isolate these terms we apply the identity $A B C-1=$ $(A-1)(B-1)(C-1)+(A-1)(B-1)+(A-1)(C-1)+(B-1)(C-1)+(A-1)+(B-1)+(C-1)$ to Eq. (29). Only terms involving just one of the three $F$-functions can contribute to the delta function, so we find that for $\nu, q>0$ the intensities may be written

$$
\begin{aligned}
& I_{C D E}(\tilde{\Omega})=W_{\rho}(\tilde{\Omega}) \delta\left(\nu-v_{\rho} q\right)+\ldots \\
& I_{S P E}(\tilde{\Omega})=\left(\sum_{s_{1} s_{2}= \pm 1} s_{1} s_{2} W_{\sigma}^{s_{1}, s_{2}}(\tilde{\Omega})\right) \delta\left(\nu-v_{\sigma} q\right)+\ldots
\end{aligned}
$$

where the ellipses denote the continuum terms and the delta function coefficients are, respectively:

$$
\begin{gathered}
W_{\rho}(q, \tilde{\Omega})=8 \pi \int_{0}^{\infty} d t_{1} f_{r_{1} s_{1}}\left(t_{1}\right) \int_{0}^{\infty} d t_{2} f_{r_{2} s_{2}}^{\star}\left(t_{2}\right) \int_{-\infty}^{\infty} d R^{\prime} e^{-i q R^{\prime}}\left\{\left[F_{1,1}^{\rho}\left(R^{\prime} ; t_{1}, t_{2}\right)\right]^{\alpha_{\rho}}-1\right\} \\
W_{\sigma}^{s_{1} s_{2}}(q, \tilde{\Omega})=2 \pi \int_{0}^{\infty} d t_{1} f_{r_{1} s_{1}}\left(t_{1}\right) \int_{0}^{\infty} d t_{2} f_{r_{2} s_{2}}^{\star}\left(t_{2}\right) \int_{-\infty}^{\infty} d R^{\prime} e^{-i r_{1} q R^{\prime}}\left\{\left[F_{1,1}^{\sigma}\left(R ; t_{1}, t_{2}\right)\right]^{\frac{s_{1} s_{2}}{2}}-1\right\} .
\end{gathered}
$$

These formulae have an involved analytic structure, which includes a discontinuity when either the incoming or the outgoing laser frequency is precisely in resonance with the valence band to conduction band Fermi level energy difference. For large frequencies, the correct asymptotic behavior arises from cancellation among oscillating terms. To make the structure manifest and obtain forms which are convenient for numerical evaluation we employ the further 
series of transformations given in the Appendix, leading for $|\tilde{\Omega}|>v_{\rho, \sigma} q / 2$ to

$$
\begin{aligned}
W_{\rho}(q,|\tilde{\Omega}|> & \left.v_{\rho} q / 2\right)=\frac{2^{\frac{3}{2}+\alpha} \sin \left(\frac{\pi(1+\alpha)}{2}\right) \Gamma(1-2 \alpha)}{\tilde{\Omega}^{1-2 \alpha}} \int_{0}^{\pi / 4} d \phi \int_{0}^{\pi / 2} d \theta \frac{\cos ^{2} \theta(\cot \theta)^{\alpha}}{\sqrt{1+\cos 2 \phi \cos 2 \theta}} \times \\
& \left(\left(\frac{1}{\left(\cos \phi-\frac{v_{\rho} q}{2 \tilde{\Omega}} \sqrt{\frac{1+\cos 2 \phi \cos 2 \theta}{2}}\right)}\right)\right. \\
W_{\sigma}\left(q,|\tilde{\Omega}|>v_{\sigma} q / 2\right)=\frac{2^{\frac{1}{2}+\alpha} \Gamma(1-2 \alpha)}{\tilde{\Omega}^{1-2 \alpha}} \int_{0}^{\pi / 4} d \phi \int_{0}^{\pi / 2} & \left.d \theta \frac{\cos 2 \theta}{\sqrt{1+\cos 2 \phi \cos 2 \theta} \times}\right) \\
& \left(\left(\frac{1}{\left(\cos \phi+\frac{v_{r} h o q}{2 \tilde{\Omega}} \sqrt{\frac{1+\cos 2 \phi \cos 2 \theta}{2}}\right)}\right)\right. \\
& \left.\left.\left(\frac{1}{\left(\cos \phi-\frac{v_{\sigma} q}{2 \Omega} \sqrt{\frac{1+\cos 2 \phi \cos 2 \theta}{2}}\right.}\right)\right)^{1-2 \alpha}-\left(\frac{1}{\left(\cos \phi+\frac{v_{\sigma} q}{2 \Omega} \sqrt{\frac{1+\cos 2 \phi \cos 2 \theta}{2}}\right)}\right)^{1-2 \alpha}\right)
\end{aligned}
$$

For $|\tilde{\Omega}|<v_{\rho, \sigma} q / 2$ we obtain

$$
\begin{aligned}
W_{\rho}(q,|\tilde{\Omega}| & \left.<v_{\rho} q / 2\right)=\frac{2^{\frac{5}{2}-\alpha}}{\left(v_{\rho} q\right)^{1-2 \alpha}} \sin \left(\pi \frac{1+\alpha}{2}\right) \Gamma(1-2 \alpha) \int_{0}^{\pi / 4} d \phi \int_{0}^{\pi / 2} d \theta \frac{\cos ^{2} \theta(\cot \theta)^{\alpha}}{\sqrt{1-\cos 2 \phi \cos 2 \theta}} \times \\
& \left(\frac{1}{\left(\sqrt{\frac{1-\cos 2 \phi \cos 2 \theta}{2}}+\frac{2 \tilde{\Omega}}{v_{\rho} q} \sin \phi\right)^{1-2 \alpha}}+\frac{1}{\left(\sqrt{\frac{1-\cos 2 \phi \cos 2 \theta}{2}}-\frac{2 \tilde{\Omega}}{v_{\rho} q} \sin \phi\right)^{1-2 \alpha}}\right)
\end{aligned}
$$

and

$$
\begin{aligned}
W_{\sigma}(q,|\tilde{\Omega}| & \left.<v_{\sigma} q / 2\right)=\frac{2^{\frac{3}{2}-\alpha}}{\left(v_{\sigma} q\right)^{1-2 \alpha}} \Gamma(1-2 \alpha) \int_{0}^{\pi / 4} d \phi \int_{0}^{\pi / 2} d \theta \frac{\cos 2 \theta}{\sqrt{1-\cos 2 \phi \cos 2 \theta}} \times \\
& \left(\frac{1}{\left(\sqrt{\frac{1-\cos 2 \phi \cos 2 \theta}{2}}+\frac{2 \tilde{\Omega}}{v_{\sigma} q} \sin \phi\right)^{1-2 \alpha}}-\frac{1}{\left(\sqrt{\frac{1-\cos 2 \phi \cos 2 \theta}{2}}-\frac{2 \tilde{\Omega}}{v_{\sigma} q} \sin \phi\right)^{1-2 \alpha}}\right) .
\end{aligned}
$$

The change in analytic structure occurring at $|\tilde{\Omega}|=v_{\rho, \sigma} q / 2$ is manifest in these expressions by the factor of $\sqrt{1 \pm \cos 2 \phi \cos 2 \theta}$ in the denominator. In the $|\tilde{\Omega}|<v_{\rho, \sigma} q / 2$ regime $\sqrt{1-\cos 2 \phi \cos 2 \theta}$ and the $\left(\sqrt{\frac{1-\cos 2 \phi \cos 2 \theta}{2}} \pm \frac{2 \tilde{\Omega}}{v_{\rho, \sigma} q} \sin \phi\right)^{1-2 \alpha}$ have singularities at the same point, leading to a strong (but still integrable) singularity at $\phi=\theta=0$, which is absent for $|\tilde{\Omega}|>v_{\rho, \sigma} q / 2$. To understand the singularity one may expand for small $\phi, \theta$, finding the leading behavior (see the Appendix)

$$
W_{\rho, \sigma} \sim \frac{1}{\alpha} J_{\alpha}\left(\frac{2 \tilde{\Omega}}{v_{\rho, \sigma} q}\right) \quad\left(|\tilde{\Omega}|<v_{\rho, \sigma} q / 2\right)
$$

with $J_{\alpha}$ a function with an additional integrable divergence at $|\tilde{\Omega}|=v_{\rho, \sigma} q / 2$. The extra contribution arising from Eq. (39) leads to steps in the CDE and SPE intensities as the frequency is moved across resonance, with magnitude diverging in the non-interacting limit $\alpha \rightarrow 0$.

Fig. 4 shows, on a logarithmic scale the CDE and SPE results calculated from Eqs. (35)-(38). For clarity of presentation we have removed the prefactors $\left(\left(\Gamma(1-2 \alpha) /\left(v_{\rho, \sigma} q\right)^{1-2 \alpha}\right.\right.$ and have expressed the dependence on laser 
frequency via the ratio of the laser frequency measured from resonance $\tilde{\Omega}$ to the mode frequency $\nu$ (note that at fixed momentum transfer $q$ the SPE and CDE mode frequencies will differ). Far from resonance, the CDE intensity is seen to be much larger than the SPE intensity, but close to resonance, the two are comparable. The main panel of Fig. [5 shows the ratio of the SPE to the CDE absorption. We see that the ratio is only appreciable if the incident photon energy is essentially 'on resonance'. The ratio exhibits a derivative discontinuity (seen more clearly in the inset) at $|\Omega|=\nu / 2$ (here $\nu=v_{\rho, \sigma} q$ represents the resonance energy for charge and spin modes respectively); the on-resonance ratio is about 0.5 independent of the Luttinger exponent. (Of course the factors of velocity which have been removed from the results will lead to an additional dependence on $\alpha$ ).

\section{RPA CALCULATION}

In order to compare our calculation to previous literature we present the analogue, for the model considered here, of the "Fermi Liquid' calculation discussed extensively in previous work $15,16,17,18,23,30$. The previous calculations neglect backscattering entirely, and treat the forward scattering part of the interaction in the RPA approximation; we make the same approximations here. We obtain analytical expressions apparently not given in previous literature. We consider here a model with short ranged interaction, parametrized by a constant amplitude $V$. The CDE feature is then a zero-sound collective mode with a velocity shifted from the Fermi velocity by the interaction strength.

We evaluate the diagrams shown in Fig [6 The analytic expression corresponding to the diagram labelled 1 is

$$
D 1=-T \sum_{\omega_{n}} \int \frac{d p}{2 \pi} G_{d}^{2}\left(i \Omega+i \nu / 2+i \omega_{n}, p+q / 2\right) G_{c}\left(p+q, i \omega_{n}+i \nu\right) G_{c}\left(p, i \omega_{n}\right)
$$

Setting the valence band velocity to zero, analytically continuing on the laser frequency $\Omega$ and measuring it from resonance and performing the frequency sum yields

$$
D 1=-\int \frac{d p}{2 \pi} \frac{1}{i \nu+\varepsilon_{p}-\varepsilon_{p+q}}\left(\frac{f_{p}-1}{\left(\tilde{\Omega}+i \nu / 2+\varepsilon_{p}\right)^{2}}-\frac{f_{p+q}-1}{\left(\tilde{\Omega}-i \nu / 2+\varepsilon_{p+q}\right)^{2}}\right),
$$

where $f_{p}$ is Fermi distribution function. We next linearize the conduction band dispersion, perform the $p$ integral (bearing in mind that we must take the principal value), drop terms of order $v q / E_{F}$ and analytically continue on $\nu$. Unlike the Luttinger liquid expressions obtained in previous sections, this expression as it stands is infinite in the range $|\tilde{\Omega}|<\nu q / 2$. Previous work ${ }^{23.30}$ resolved this divergence by introducing a phenomenological broadening parametrized by a quantity $\Lambda$. We follow this procedure here, but emphasize that because the behavior in the region $|\tilde{\Omega}|<\nu / 2$ is determined entirely by this phenomenological parameter, our results in this region have, strictly speaking, no meaning. We obtain

$$
D 1=\Pi(\nu, q)\left(\frac{\tan ^{-1}\left(\frac{\tilde{\Omega}+\nu / 2}{\Lambda}\right)-\tan ^{-1}\left(\frac{\tilde{\Omega}-\nu / 2}{\Lambda}\right)}{\Lambda}\right)
$$

Here

$$
\Pi(\nu, q)=\frac{1}{2 \pi v_{F}}\left(\frac{1}{\nu-v_{F} q-i \delta}+\frac{1}{\nu+v_{F} q-i \delta}\right)=\frac{\nu}{\pi v_{F}} \frac{1}{\nu^{2}-v_{F}^{2} q^{2}}
$$

The analytical expression corresponding to the diagram labelled 2 is

$$
D 2=(D 22)^{2} \frac{V}{1+\chi V}
$$

with

$$
\begin{aligned}
D 22 & =-T \sum_{\omega_{n}} \int \frac{d p}{2 \pi} G_{d}\left(i \Omega+i \nu / 2+i \omega_{n}, p+q / 2\right) G_{c}\left(p+q, i \omega_{n}+i \nu\right) G_{c}\left(p, i \omega_{n}\right) \\
\chi & =T \sum_{\omega_{n}} \int \frac{d p}{2 \pi} G_{c}\left(p+q, i \omega_{n}+i \nu\right) G_{c}\left(p, i \omega_{n}\right) \\
& =\frac{\left(v_{F} q\right)^{2}}{\pi v_{F}} \frac{1}{\nu^{2}-v_{F}^{2} q^{2}}
\end{aligned}
$$


The arguments leading to Eq. (42) may be repeated here. The divergence is weaker; indeed the obtained expressions are finite everywhere except at $|\tilde{\Omega}|=\nu / 2$, where there is a logarithmic divergence. Introducing the same broadening as above yields

$$
\begin{aligned}
D 22 & =-\int \frac{d p}{2 \pi} \frac{1}{i \nu+\varepsilon_{p}-\varepsilon_{p+q}}\left(\frac{f_{p}-1}{\left(\tilde{\Omega}+i \nu / 2+\varepsilon_{p}\right)}-\frac{f_{p+q}-1}{\left(\tilde{\Omega}-i \nu / 2+\varepsilon_{p+q}\right)}\right) \\
& =\Pi(q, \nu) \frac{1}{2} \ln \left[\frac{(\tilde{\Omega}+\nu / 2)^{2}+\Lambda^{2}}{(\tilde{\Omega}-\nu / 2)^{2}+\Lambda^{2}}\right]
\end{aligned}
$$

The total unpolarized RRS cross section, in this approximation, is thus

$$
\begin{aligned}
I_{R R S} & =\Pi(q, \nu)\left(\left(\frac{\tan ^{-1}\left(\frac{\tilde{\Omega}+\nu / 2}{\Lambda}\right)-\tan ^{-1}\left(\frac{\tilde{\Omega}-\nu / 2}{\Lambda}\right)}{\Lambda}\right)+\frac{\frac{1}{4} \ln ^{2}\left[\frac{(\tilde{\Omega}+\nu / 2)^{2}+\Lambda^{2}}{(\tilde{\Omega}-\nu / 2)^{2}+\Lambda^{2}}\right] V \Pi}{1+\chi V}\right) \\
& =\frac{1}{\pi v_{F}} \frac{\nu}{\nu^{2}-v_{F}^{2} q^{2}}\left(\left(\frac{\tan ^{-1}\left(\frac{\tilde{\Omega}+\nu / 2}{\Lambda}\right)-\tan ^{-1}\left(\frac{\tilde{\Omega}-\nu / 2}{\Lambda}\right)}{\Lambda}\right)+\frac{\nu V}{\pi v_{F}} \frac{\frac{1}{4} \ln ^{2}\left[\frac{(\tilde{\Omega}+\nu / 2)^{2}+\Lambda^{2}}{(\tilde{\Omega}-\nu / 2)^{2}+\Lambda^{2}}\right]}{\nu^{2}-v_{\rho}^{2} q^{2}}\right)
\end{aligned}
$$

with $v_{\rho}=v_{F} \sqrt{1+\frac{V}{\pi v_{F}}}$. Observe that if the phenomenological broadening parameter $\Lambda$ is set to 0 the RPA approximation to the RRS intensity diverges when $\tilde{\Omega}= \pm \nu / 2$, in other words when the incoming or the outgoing photon is in resonance with a transition of the system.

From these formulae the SPE and CDE intensities may easily be obtained. We have

$$
\begin{aligned}
& I_{C D E}=\frac{1}{8 v_{F} \nu}\left(\frac{v_{\rho}}{v_{F}}\right)^{2} \ln ^{2}\left[\frac{(\tilde{\Omega}+\nu / 2)^{2}+\Lambda^{2}}{(\tilde{\Omega}-\nu / 2)^{2}+\Lambda^{2}}\right] \delta\left(\nu-q v_{\rho}\right) \\
& I_{S P E}=\frac{1}{2 v_{F}}\left[\left(\frac{\tan ^{-1}\left(\frac{\tilde{\Omega}+\nu / 2}{\Lambda}\right)-\tan ^{-1}\left(\frac{\tilde{\Omega}-\nu / 2}{\Lambda}\right)}{\Lambda}\right)-\frac{1}{4 \nu} \ln ^{2}\left[\frac{(\tilde{\Omega}+\nu / 2)^{2}+\Lambda^{2}}{(\tilde{\Omega}-\nu / 2)^{2}+\Lambda^{2}}\right]\right] \delta\left(\nu-q v_{F}\right)
\end{aligned}
$$

The CDE/SPE ratio is plotted in the inset to Fig. 5 for $\Lambda=0.1 \nu$. We see from this inset that the ratio is generically very small (smaller than the corresponding ratio in a Luttinger liquid), but that for all $|\tilde{\Omega}|<v_{\rho, \sigma} q / 2$, the divergence of the SPE term (cut-off here by a small value of the phenomenological parameter $\Lambda$ ), yields a large value of the ratio.

\section{DISCUSSION}

\section{A. Overview of calculation}

In this paper we have presented a complete analytical theory of Resonant Raman Scattering in Luttinger liquids, and for comparison we have also given the analogous results for what is referred to in the literature as the 'Fermi liquid' approximation. In this section we discuss the implications of our results. In this first subsection we outline the essential assumptions underlying the calculation. In the second subsection we discuss the relevant parameter regimes and the different behavior expected in each regime, and in the third subsection we discuss the extent to which Raman scattering has observed, or can be expected to observe, evidence for Luttinger liquid behavior in quasi one dimensional systems.

We note that both Luttinger liquid and 'Fermi Liquid' calculations require three assumptions:

(i) that the energy $\nu$ and momentum $q$ transferred to the one dimensional electron system are small in comparison the the Fermi energy and Fermi momentum of the one dimensional electron system 
(ii) that the Raman process involves excitation from a one dimensional valence band to an empty state in a one dimensional conduction band, followed by decay of an electron from a filled state in the conduction band into the hole state in the valence band

(iii) that 'excitonic' correlations between conduction band states and the intermediate state may be neglected.

Assumption (i) is essentially a condition that the experimental resolution is sufficient to reveal the low energy physics of the system of interest. From the theoretical point of view it could easily be relaxed at the expense of introducing a more complicated description of the conduction band.

Assumption (ii) is clearly applicable to strictly one dimensional systems such as carbon nanotubes (where the valence band is clearly one dimensional) but may not be applicable to quantum wire structures created by lithographic or MBE techniques on a three dimensional substrate. In this situation, valence band carriers may be able to move transverse to the wire. Because momentum transverse to the wire is not conserved in the optical absorbtion process, in this case the function $\phi(x, t)$ in Eq. (11) must be integrated over a range of transverse momenta, leading to a broadening of the resonance. However, assumption (ii) may also be relaxed. The key issue is that the intermediate state (of whatever origin) disperses only along the wire.

The crucial assumption is (iii), neglect of excitonic correlations. These are likely to play a crucial role in the QWR structures, keeping the valence hole near the wire and therefore allowing a sharp resonance to occur. Neglect of excitonic correlations entered the theory at several points, and the theory cannot be trivially modified to include them. Construction of a theory including excitonic effects is an important open issue in the field.

By combining the three assumptions given above with standard second order perturbation methods, we obtained results, presented in Sec. [V] and Eqs. (35)-(38), for the resonant Raman scattering intensities of a one dimensional electronic system described by the Luttinger liquid model. For comparison, we also presented analytical expressions for the Raman intensities of the previously discussed 'Fermi liquid model" (in which the only electron-electron interaction considered is forward scattering in the density channel, and this is treated in the random phase approximation (RPA)). In the far-from resonance regime, we showed that a perturbative treatment could be applied, and this was used to provide a detailed characterization of the different features of the spectrum, including 'CDE' and 'SPE' peaks and a continuum absorption and the dependence of the intensity of these features on the difference of the laser frequency from resonance. We also obtained simple, easily evaluated expressions for the integrated intensities in the SPE and CDE peaks for all values of the laser frequency, and from these we obtained results for the ratio of SPE to CDE intensities. The expressions have an interesting analytical structure in the near-resonance region, which is discussed below.

\section{B. Physics of RRS spectrum-difference between Raman spectra of Luttinger and Fermi Liquids}

The Raman process creates a particle and a hole in the conduction band, at a space-time separation controlled by the difference of the laser frequency from a resonance condition. The particle-hole pair is in general not an eigenstate of the conduction band Hamiltonian, but decays into eigenstates. The important question, therefore, is "what properties of the eigenstates are reveals by Raman scattering". In the one dimensional context one may sharpen this question to "what aspects of the Raman spectrum reveal characteristic features of the Luttinger liquid physics expected to occur in one dimensional systems?". In order to answer this question precisely, it is necessary to discuss the eigenstates of one dimensional electronic systems.

We are concerned here with polarized channel Raman scattering in systems with negligible spin-orbit coupling and therefore must consider excitations which do not change the total spin of the one dimensional system. In a Fermi liquid, the eigenstates are particle-hole pairs, either organized into collective models or existing as incoherent "particle-hole continuum' states. Further, in a Fermi liquid at low energies and long wavelengths one may restrict attention to states consisting of a single excited (quasi)-particle or (quasi)-hole: multipair states are important only as virtual processes renormalizing matrix elements and dispersions. In a Fermi liquid with repulsive interactions, there are two kinds of relevant states. One is the zero sound (or 'plasmon') mode, referred to in the Raman literature as the 'CDE' mode. (In principal other collective modes exist; these are rarely important in practice). The CDE excitation typically has a well defined energy-momentum dispersion relation $\nu=v_{\rho}(q)|q|$ with velocity $v_{\rho}(q)$ greater than the Fermi velocity $v_{F}$.

The other class of states, referred to in the Raman literature as 'SPE', are the particle-hole continuum states. In two and three dimensional systems, these excitations exist in the range $0 \leq \nu \leq v_{F} q$; in $d=1$ they exist only in the range $v_{F}|q|-\frac{v_{F} q^{2}}{2 p_{0}} \leq|\nu| \leq v_{F}|q|+\frac{v_{F} q^{2}}{2 p_{0}}$ with $p_{0}=\frac{v_{F}}{\partial^{2} \varepsilon_{p} /\left.\partial p^{2}\right|_{p=p_{F}}}$ a measure of the curvature of the quasi-particle dispersion. In any dimension, the 'SPE' or continuum excitations make an important contribution to the specific heat and to most response functions. However, the contribution of the continuum excitations to the structure factor (density response function) is typically much smaller than that of the CDE (plasmon) mode. In $d>1$ the CDE contribution to the structure factor is larger than the SPE contribution by a factor of the dimensionless long wavelength interaction 
strength, which for a charged Fermi liquid involves factors of $\left(q_{T F} / q\right)^{d-1}$ arising from the long ranged nature of the Coulomb interaction (here $q_{T F}$ is the Thomas-Fermi screening length). In $d=1$ the factor arising from the Coulomb interaction is only logarithmic; however the special kinematics of one dimensional systems lead to additional constraints. Within the RPA approximation the SPE contribution to the structure factor is smaller than the CDE contribution by a factor of $\left(q / p_{0}\right)^{2}$. In the linearized dispersion limit $\left(p_{0} \rightarrow \infty\right)$ the SPE contribution to the $d=1$ structure factor vanishes entirely within RPA approximation, and indeed this vanishing may be shown via a Ward Identity $^{38}$ to occur to all orders in the interaction. We emphasize, however, that in the Fermi liquid approximation to the one dimensional electron gas, the SPE excitations exist, and may be revealed either by considering response functions other than the structure factor, or from the specific heat. A specific example of a response function other than the structure factor is provided by the Raman intensity near resonance.

In one dimensional systems, the Fermi liquid approximation is believed to provide a very poor description of the low energy physics, which is believed to be more accurately represented by the Luttinger liquid model. Consider first a spinless electron gas. This could be realized in practice by applying a magnetic field large enough to fully spin-polarize the conduction band. It is believed that a correct treatment (for example, via the Luttinger model) would predict that the only elementary excitation is a boson, roughly equivalent to the zero sound or plasmon mode. Thus in the spinless Luttinger liquid case, in all response functions and also in the specific heat, only superpositions of plasmons would be observed, whereas the 'fermi liquid' approximation predicts one would observe features at both energies. In Raman language, in the Luttinger liquid case only the CDE mode would be visible, no matter how closely the system is tuned to resonance, whereas for a hypothetical 1D Fermi liquid, excitation at the SPE energy would become visible as the laser frequency is tuned to resonance.

Now consider the Luttinger liquid formed by the usual electron gas, in zero magnetic field. In this case, two classes of elementary excitation exist: charge and spin bosons. States of total spin zero may be constructed from states of two or more spin bosons, even if no charge bosons are present, and these may make important contributions to many response functions and to the specific heat. In the linearized dispersion limit, states involving only spin bosons do not appear in the structure function, which couples only to charge. However, curvature in the dispersion leads to charge-spin coupling and in particular to terms allowing a virtual charge boson to decay into two spin bosons. Thus, just as in the Fermi liquid case, if nonlinearity in the underlying dispersion is neglected, only the CDE mode is visible in the structure factor, but at order $\left(q / p_{0}\right)^{2}$ SPE contributions appear, and of course SPE contributions appear in response functions other than the structure factor.

To summarize this subsection: as long as the electron number is not changed, the apparently profound differences between a Luttinger and a Fermi liquid produce only minor, quantitative differences in the excitation spectrum of an electron gas in zero magnetic field. In both cases, one has two classes of excitation, which may be labelled CDE and SPE. Far from resonance, the matrix element determining the Raman spectrum is the density operator $\rho_{q, \nu}$ plus additional operators which vanish as the ratio $\nu / \tilde{\Omega}$ vanishes (i.e. as the laser frequency is moved arbitrarily far from the resonance condition). In the limit $\nu / \tilde{\Omega} \rightarrow 0$ the Raman spectrum is the same as the structure factor. In both Luttinger and Fermi liquids the SPE mode makes an 'intrinsic' contribution to the structure factor which is smaller by a factor of $\left(q / p_{0}\right)^{2}$ independent of difference of laser frequency from resonance and which vanishes when curvature is neglected. However, for a spin polarized electron gas, there is an important difference between a Luttinger and a Fermi liquid: in the Fermi liquid approximation an SPE branch of excitations exists, whereas in a Luttinger liquid it does not.

\section{Approach to resonance}

The previous subsections have shown that in the polarized channel of RRS experiment (i.e. no spin-flipping of final electron configuration), the differences between the predicted Raman spectra of a Luttinger liquid and Fermi liquid approximations to the one dimensional electron gas are quantitative, not qualitative. Very far from resonance, the Raman spectrum is determined by the structure factor, so differences between the two cases involve differences in the structure factor. These are unimportant, arising from different coefficients of the $\left(q / p_{0}\right)^{2}$ terms in the structure factor. Because the coefficients involve non-universal factors, this difference does not provide a useful distinction between the two cases. A more useful distinction arises from consideration of the changes arising in the spectrum as the laser frequency is brought closer to a resonance condition. As this occurs, operators other than the structure factor begin to contribute to the Raman spectrum, and the nature and coefficients of these operators may be used to distinguish between the two cases.

In discussing the near-resonance behavior it is helpful to refer to Fig. 6 which shows the diagrams commonly considered in the "Fermi liquid" treatment of the RRS process. From these diagrams one sees that in the 'Fermi liquid' approximation, the states created by the Raman process have nonvanishing overlap with the two sorts of exact eigenstates (CDE and SPE) of the system. Diagram 1 gives the dominant contribution to the probability of creating 
an SPE excitation. The direct overlap between the state created by the Raman process and the exact eigenstate of the system means that in the absence of 'excitonic' correlations between the particle-hole pair and the intermediate resonant state (sketched as dash-dot lines in diagram 1 of Fig (6), this diagram diverges strongly. (The diagram might also diverge strongly even in the presence of excitonic effects: this point has not been investigated.) Indeed one sees from Eq. (42) that very near resonance the term diverges as $(\tilde{\Omega} \pm \nu / 2)^{-1}$ and (in the absence of excitonic correlations or of the phenomenological broadening $\Lambda$ ) is infinite for $|\tilde{\Omega}|<\nu / 2$. On the other hand, one sees from diagram 2 that the coupling of the Raman process to the CDE excitation goes through a range of virtual states (represented by the triangles in diagram 2); this broadens the resonance effect so that the divergence as resonance is approached is only logarithmic and the result is finite for $|\tilde{\Omega}|<\nu / 2$. However, the one dimensional kinematics lead for $|\tilde{\Omega}|>\nu / 2$ to a much greater CDE amplitude than an SPE amplitude, so that the SPE/CDE ratio only becomes of order unity very close to the resonance condition. Further, for $|\tilde{\Omega}|<\nu / 2$ the Fermi liquid approximation to the SPE intensity is, strictly speaking, infinite, and must be regularized by consideration of processes so far omitted from the approximation. At present there is no theory of this regularization, which is parametrized by a phenomenological broadening $\Lambda$. The 'Fermi liquid' results presented in this paper in the near-resonance regime are probably meaningless. We suspect that this issue also arises in the higher dimensional calculations performed so far.

In the Luttinger liquid approximation, the particle-hole pair created in the Raman process has vanishing overlap with any exact eigenstate. This has three consequences: first, a continuum absorption arising from multiboson excitations is predicted to lie in between the SPE and CDE peaks (actually, this continuum would exist also in a Fermi liquid, but would be much weaker). Also, away from resonance, the SPE/CDE ratio is larger than in the Fermi liquid approximation, and it varies with a power related to the Luttinger liquid exponent $\alpha$. Finally, in the on-resonance regime $|\tilde{\Omega}|<\nu / 2$ both SPE and CDE contributions are predicted to be finite, although both exhibit a discontinuity when $|\tilde{\Omega}|=\nu / 2$. Fermi liquid behavior is recovered because the magnitude of the discontinuity is $\alpha^{-1}$ and diverges in the noninteracting $(\alpha \rightarrow 0)$ limit.

We suggest that in the on-resonance regime $|\tilde{\Omega}| \sim \nu / 2$ neither the Fermi liquid nor the Luttinger liquid calculation are likely to be quantitatively reliable, because excitonic correlations (neglected here) are likely to be important: near resonance the intermediate state lasts so long that it must interact with the conduction band excitations, and these interactions are likely to have a nontrivial interplay with the non-analyticities arising when $|\tilde{\Omega}|=\nu / 2$.

\section{SUMMARY}

In this paper we have used the full Luttinger liquid model to analytically and numerically calculate the RRS spectrum for both polarized and depolarized spectroscopy, and have presented for comparison a 'Fermi liquid' calculation. We obtained results for both a short ranged (screened Coulomb) interactions and for the more physically relevant case of the long ranged Coulomb interaction. We clarified the difference between the 'Fermi-liquid' and Luttinger liquid results, and argued that proper treatment of excitonic interactions (neglected in all treatments so far) is essential for obtaining reliable results. Our results in Fig. 2 and Fig. 3 shows that the RRS spectra of both short-ranged and long-ranged interactions are qualitatively similar, except that in the unscreened Coulomb case the momentum dependent charge velocity makes the phase space of multiboson excitations to be highly restricted, which shift the multiboson excitations to higher energy than the plasmon energy $\omega=q v_{q}$, causing such unusual broadening. The SPE-CDE ratio is generically larger in a Luttinger liquid than in a Fermi liquid, except on-resonance, where excitonic effects probably invalidate either calculation.

\section{ACKNOWLEDGMENT}

We thank useful discussion with A. Pinczuk about the details of Raman scattering experiments. This work is supported (DWW and SDS) by US-ONR, US-ARO, and DARPA, and by NSF-DMR-0338376 (AJM). 


\section{APPENDIX A: EVALUATION OF DELTA FUNCTION CONTRIBUTIONS}

\section{A. Overview}

The Appendix presents the additional transformations needed to put Eqs. (33)-(34) into forms convenient for further discussion and numerical evaluation. We have to deal with an expression of the form (note that for simplicity, we define $\Omega$ to be the light frequency with respect to the resonance energy throughout this Appendix.)

$$
W=\int_{0}^{\infty} \frac{d t_{1} e^{i(\Omega+i \varepsilon) t_{1}}}{t_{1}^{1+\alpha}} \int_{0}^{\infty} \frac{d t_{2} e^{-i(\Omega-i \varepsilon) t_{2}}}{t_{2}^{1+\alpha}} I\left(t_{1}, t_{2}\right)
$$

with $\varepsilon \rightarrow 0^{+}$being an infinitesmall converging factor and

$$
I\left(t_{1}, t_{2}\right)=\int_{-\infty}^{\infty} d R e^{-i q R} F\left(t_{1}, t_{2}, R\right)
$$

and $F$ given by

$$
F=\left(\frac{\frac{v^{2}\left(t_{1}+t_{2}\right)^{2}}{4}-(R+i \varepsilon)^{2}}{\frac{v^{2}\left(t_{1}-t_{2}\right)^{2}}{4}-(R+i \varepsilon)^{2}}\right)^{\beta}-1,
$$

where for the charge case $v=v_{\rho}$ and $\beta=(1+\alpha) / 2$, while in the spin case, $v=v_{\sigma}=v_{F}$ and $\beta=1 / 2$. As in Sec. III] we have assumed that the valence band velocity is much smaller than the Fermi velocity in the conduction band. The analytic structure of the $F$ function means that we can write (note we have also rescaled $R$ )

$$
I\left(t_{1}, t_{2}\right)=2 \sin (\pi \beta) \int_{A}^{B} d R \quad \sin \left(\frac{q R}{2}\right) F_{2}(B, A, R)
$$

with

$$
F_{2}\left(t_{1}, t_{2}, R\right)=\left(\frac{B^{2}-R^{2}}{R^{2}-A^{2}}\right)^{\beta}
$$

and $B=t_{1}+t_{2}$ and $A=\left|t_{1}-t_{2}\right|$.

By making the following changes of variables: $R \rightarrow \sqrt{u} ; u \rightarrow v+A^{2} ; v \rightarrow \sqrt{B^{2}-A^{2}} \sin \theta$ and restoring the explicit forms of $B$ and $A$ we obtain

$$
I\left(t_{1}, t_{2}\right)=2 \sin (\pi \beta) t_{1} t_{2} \int_{0}^{\pi / 2} d \theta \frac{(\cos \theta)^{1+2 \beta}(\sin \theta)^{1-2 \beta} \sin \frac{v q}{2} \sqrt{t_{1}^{2}+t_{2}^{2}-2 t_{1} t_{2} \cos 2 \theta}}{\sqrt{t_{1}^{2}+t_{2}^{2}-2 t_{1} t_{2} \cos 2 \theta}}
$$

\section{B. Evaluation, large $\Omega$}

If $\Omega>\frac{v q}{2}$ then it is convenient to rotate the $t$ integrals via

$$
\begin{aligned}
& t_{1} \rightarrow i \tau_{1} \\
& t_{2} \rightarrow-i \tau_{2}
\end{aligned}
$$

getting

$$
W=\int_{0}^{\infty} \frac{d \tau_{1} e^{-\Omega \tau_{1}}}{\tau_{1}^{1+\alpha}} \int_{0}^{\infty} \frac{d \tau_{2} e^{-\Omega \tau_{2}}}{\tau_{2}^{1+\alpha}} I\left(\tau_{1}, \tau_{2}\right)
$$

with

$$
I\left(\tau_{1}, \tau_{2}\right)=2 \sin (\pi \beta) \tau_{1} \tau_{2} \int_{0}^{\pi / 2} d \theta \frac{(\cos \theta)^{1+2 \beta}(\sin \theta)^{1-2 \beta} \sinh \frac{v q}{2} \sqrt{\tau_{1}^{2}+\tau_{2}^{2}+2 \tau_{1} \tau_{2} \cos 2 \theta}}{\sqrt{\tau_{1}^{2}+\tau_{2}^{2}+2 \tau_{1} \tau_{2} \cos 2 \theta}}
$$


It is then useful to define

$$
\begin{aligned}
& \tau_{1}=\tau \cos \phi \\
& \tau_{2}=\tau \sin \phi
\end{aligned}
$$

so that

$$
W=2 \sin (\pi \beta) \int_{0}^{\pi / 2} d \phi \int_{0}^{\pi / 2} d \theta \int_{0}^{\infty} \frac{d \tau}{\tau^{2 \alpha}} e^{-\Omega \tau(\cos \phi+\sin \phi)} \frac{(\cos \theta)^{1+2 \beta}(\sin \theta)^{1-2 \beta} \sinh \frac{v q \tau}{2} \sqrt{1+\sin 2 \phi \cos 2 \theta}}{\sqrt{1+\sin 2 \phi \cos 2 \theta}}
$$

The $\tau$ integral may now be done. It is convenient to shift the origin of the $\phi$ integral by $\pi / 4$ and symmetrize in $\phi$ and to combine the two terms arising in the spin term, getting

$$
\begin{aligned}
& W_{\rho}=\frac{2^{\frac{3}{2}+\alpha} \sin \left(\frac{\pi(1+\alpha)}{2}\right) \Gamma(1-2 \alpha)}{\Omega^{1-2 \alpha}} \int_{0}^{\pi / 4} d \phi \int_{0}^{\pi / 2} d \theta \frac{\cos ^{2} \theta(\cot \theta)^{\alpha}}{\sqrt{1+\cos 2 \phi \cos 2 \theta}} \times \\
& \left(\left(\frac{1}{\left(\cos \phi-\frac{v_{\rho} q}{2 \Omega} \sqrt{\frac{1+\cos 2 \phi \cos 2 \theta}{2}}\right)}\right)^{1-2 \alpha}-\left(\frac{1}{\left(\cos \phi+\frac{v_{\rho} q}{2 \Omega} \sqrt{\frac{1+\cos 2 \phi \cos 2 \theta}{2}}\right)}\right)^{1-2 \alpha}\right) \\
& W_{\sigma}=\frac{2^{\frac{1}{2}+\alpha} \Gamma(1-2 \alpha)}{\Omega^{1-2 \alpha}} \int_{0}^{\pi / 4} d \phi \int_{0}^{\pi / 2} d \theta \frac{\cos 2 \theta}{\sqrt{1+\cos 2 \phi \cos 2 \theta}} \times \\
& \left(\left(\frac{1}{\left(\cos \phi-\frac{v_{F} q}{2 \Omega} \sqrt{\frac{1+\cos 2 \phi \cos 2 \theta}{2}}\right)}\right)^{1-2 \alpha}-\left(\frac{1}{\left(\cos \phi+\frac{v_{F} q}{2 \Omega} \sqrt{\frac{1+\cos 2 \phi \cos 2 \theta}{2}}\right)}\right)^{1-2 \alpha}\right)
\end{aligned}
$$

This formula has integrable singularities at $\phi=\theta=\pi / 2$ and $\theta=\pi / 2$ and is convenient for numerical evaluation.

\section{Evaluation, small $\Omega$}

If $\Omega<\frac{v q}{2}$ then the rotation cannot be made and we should instead define

$$
\begin{aligned}
& t_{1}=t \cos \phi \\
& t_{2}=t \sin \phi
\end{aligned}
$$

Transformations similar to those leading to Eq 63] then yield

$$
\begin{aligned}
W_{\rho}(\Omega) & =\frac{2^{\frac{5}{2}-\alpha}}{\left(v_{\rho} q\right)^{1-2 \alpha}} \sin \left(\pi \frac{1+\alpha}{2}\right) \Gamma(1-2 \alpha) \int_{0}^{\pi / 4} d \phi \int_{0}^{\pi / 2} d \theta \frac{\cos ^{2} \theta(\cot \theta)^{\alpha}}{\sqrt{1-\cos 2 \phi \cos 2 \theta}} \times \\
& \left(\frac{1}{\left(\sqrt{\frac{1-\cos 2 \phi \cos 2 \theta}{2}}+\frac{2 \Omega}{v_{\rho} q} \sin \phi\right)^{1-2 \alpha}}+\frac{1}{\left(\sqrt{\frac{1-\cos 2 \phi \cos 2 \theta}{2}}-\frac{2 \Omega}{v_{\rho} q} \sin \phi\right)^{1-2 \alpha}}\right)
\end{aligned}
$$

and

$$
\begin{aligned}
W_{\sigma}(\Omega) & =\frac{2^{\frac{3}{2}-\alpha}}{\left(v_{\sigma} q\right)^{1-2 \alpha}} \Gamma(1-2 \alpha) \int_{0}^{\pi / 4} d \phi \int_{0}^{\pi / 2} d \theta \frac{\cos 2 \theta}{\sqrt{1-\cos 2 \phi \cos 2 \theta}} \times \\
& \left(\frac{1}{\left(\sqrt{\frac{1-\cos 2 \phi \cos 2 \theta}{2}}+\frac{2 \Omega}{v_{\sigma} q} \sin \phi\right)^{1-2 \alpha}}-\frac{1}{\left(\sqrt{\frac{1-\cos 2 \phi \cos 2 \theta}{2}}-\frac{2 \Omega}{v_{\sigma} q} \sin \phi\right)^{1-2 \alpha}}\right)
\end{aligned}
$$


We observe that unlike Eqs. (64) and (65) these expressions have a double singularity at small $\phi, \theta$ and indeed lead to RRS spectra of quite different magnitudes. To see this point more clearly, consider the integrand, $\mathrm{I}_{c h}$ of Eq. (68) as $\phi, \theta \rightarrow 0$. We obtain

$$
I_{c h}=\left(\frac{1}{\theta}\right)^{\alpha} \frac{1}{\sqrt{2} \sqrt{\phi^{2}+\theta^{2}}}\left(\frac{1}{\left(\sqrt{\phi^{2}+\theta^{2}}+\frac{2 \Omega}{v_{\rho} q} \phi\right)^{1-2 \alpha}}+\frac{1}{\left(\sqrt{\phi^{2}+\theta^{2}}-\frac{2 \Omega}{v_{\rho} q} \phi\right)^{1-2 \alpha}}\right)
$$

Changing variables to $\theta=\zeta \cos \psi$ and $\phi=\zeta \sin \psi$ we have

$$
\int d \phi \theta d \theta I_{c h}=\int \zeta d \zeta d \psi \frac{1}{\sqrt{2} \zeta^{2-\alpha}(\cos \psi)^{\alpha}}\left(\frac{1}{\left(1+\frac{2 \Omega}{v_{\rho} q} \sin \psi\right)^{1-2 \alpha}}+\frac{1}{\left(1-\frac{2 \Omega}{v_{\rho} q} \sin \psi\right)^{1-2 \alpha}}\right)
$$

Integration over $\zeta$ gives an answer proportional to $1 / \alpha$.

1 See for example, A. Kanda, K. Tsukagoshi, Y. Aoyagi, and Y. Ootuka, Phys. Rev. Lett. 92, 036801 (2004); C. Bena, S. Vishveshwara, L. Balents, and M. P. A. Fisher, Phys. Rev. Lett. 89, 037901 (2002), and reference therein.

2 A. Georges, T. Giamarchi, and N. Sandler, Phys. Rev. B 61, 16393-16396 (2000), and reference therein.

3 F. Kassubek, C.A. Stafford, and H. Grabert, Phys. Rev. B 59, 7560-7574 (1999).

4 See for example, G. Fáth, Phys. Rev. B 68, 134445 (2003); R. M. Konik Phys. Rev. B 68, 104435 (2003), and reference therein.

5 D. S. Petrov, G. V. Shlyapnikov, and J. T. M. Walraven, Phys. Rev. Lett. 85, 3745-3749 (2000).

6 A. Schmeller, A.R. Goñi, A. Pinczuk, J.S. Weiner, J.M. Calleja, B.S. Dennis, L.N. Pfeiffer, and K.W. West, Phys. Rev. B 49, 14778 (1994).

7 See, for example, Y.C. Chang, L.L. Chang, L. Esaki, Appl. Phys. Lett. 471324 (1985); A.R. Goñi, K.W. West, A. Pinczuk, H.U. Baranger, H.L. Stormer, Appl. Phys. Lett. 611956 (1992).

8 S. Tomonaga, Prog. Theor. Phys. 5, 544 (1950).

9 J.M. Luttinger, J. Math, Phys. N.Y. 4, 1154 (1963).

10 F. D. M. Haldane, J. Phys. C, 14, 2585 (1981).

11 O.M. Auslaender, A.Yacoby, R. De Picciotto, K.W. Baldwin, L.N. Pfeiffer, K.W. West, Science 295, 825 (2002).

12 Y. Tserkovnyak, B.I. Halperin, O.M. Auslaender, and A.Yacoby, Phys. Rev. Lett. 89, 136805 (2002); ibid. Phys. Rev. B 68, 0453XX (2003).

13 H. W. Yeom, K. Horikoshi, H. M. Zhang, K. Ono, and R. I. G. Uhrberg, Phys. Rev. B 65, 241307 (2002); T. Mizokawa, K. Nakada, C. Kim, Z.-X. Shen, T. Yoshida, A. Fujimori, S. Horii, Y. Yamada, H. Ikuta, and U. Mizutani, Phys. Rev. B 65, 193101 (2002).

14 J.E. Zucker, A. Pinczuk, D.S. Chemla, and A.C. Gossard, Phys. Rev. B 35, 2892 (1987); G. Danan, A. Pinczuk, J.P. Valladares, L.N. Pfeiffer, K.W. West, and C.W. Tu, Phys. Rev. B 39, 5512-5515 (1989).

15 J. K. Jain, and P. B. Allen, Phys. Rev. Lett. 54, 947 (1985); J. K. Jain, and P. B. Allen, Phys. Rev. Lett. 54, 2437 (1985); S. Das Sarma and E. H. Hwang, Phys. Rev. Lett. 81, 4216 (1998).

16 J. K. Jain, and S. Das Sarma, Phys. Rev. B 36, 5949 (1987); J. K. Jain, and S. Das Sarma, Surf. Sci. 196, 466 (1988).

17 S. Das Sarma, Elementary Excitations in Low-Dimensional Semiconductor Structures. p. 499 in Light scattering in Semiconductor Structures and Superlattices, edited by Lockwood, D. J. and Young, J. F. (Plenum, New York, 1991).

18 D. Pines, and P. Nozieres, The Theory of Quantum Liquids (Benjamin, New York, 1966).

19 A. L. Fetter and J. D. Walecka, Quantum Theory of Many-particle Systems, (McGraw-Hill, San Francisco, 1971).

20 A. R. Goñi, A. Pinczuk, J. S. Weiner, J. M. Calleja, B. S. Dennis, L. N. Pfeiffer, and K. W. West, Phys. Rev. Lett. 67, 3298 (1991).

21 C. Schüller, G. Biese, K. Keller, C. Steinebach, and D. Heitmann, Phys. Rev. B 54, R17304 (1996).

22 A. Pinczuk, B.S. Dennis, L.N. Pfeiffer, and K.W. West, Philosophical Magazine B 70, 429 (1994) and references therein.

23 S. Das Sarma and D. W. Wang, Phys. Rev. Lett. 83, 816 (1999).

${ }^{24}$ M. Sassetti and B. Kramer, Phys. Rev. Lett. 80, 1485 (1998).

25 D. W. Wang, A. J. Millis, and S. Das Sarma, Phys. Rev. Lett. 85, 4570 (2000).

26 I. K. Marmorkos and S. Das Sarma, Phys. Rev. B 45, 13396 (1992).

27 P. A. Wolff, Phys. Rev. 171, 436 (1968); F. A. Blum, Phys. Rev. B 1, 1125 (1970).

28 H.J. Schulz, Phys. Rev. Lett. 71, 1864 (1993).

29 D.W. Wang and S. Das Sarma, Phys. Rev. B 65, 035103 (2002).

30 D.W. Wang and S. Das Sarma, Phys. Rev. B 65, 125322 (2002).

31 B. Jusserand, M. N. Vijayaraghavan, F. Laruelle, A. Cavanna, and B. Etienne, Phys. Rev. Lett. 85, 5400 (2000).

32 J. Voit, Rep. Prog. Phys., 58, 977 (1995). 


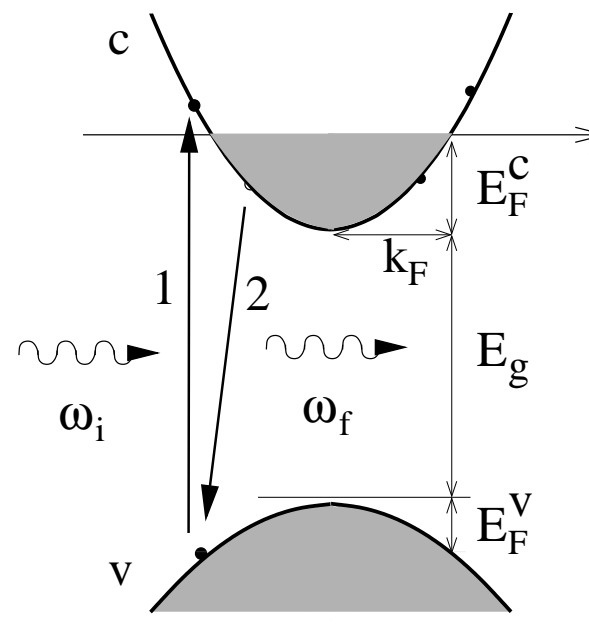

(a)

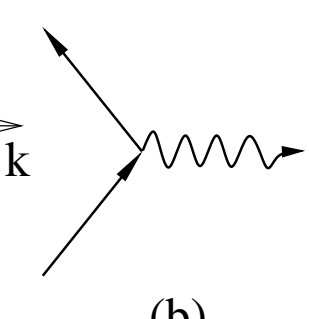

(b)

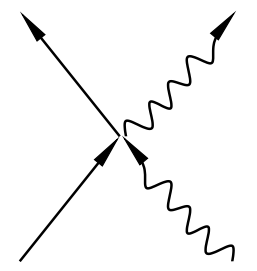

(c)

FIG. 1: (a) Schematic representation of RRS process in the direct gap two band model of electron doped GaAs nanostructures. $\omega_{i}$ and $\omega_{f}$ are the initial and final frequencies of the external photons. (b) and (c) are the three-leg and four-leg scattering vertices for electron-photon interaction.

33 P. A. Wolff, Phys. Rev. Lett. 16, 225 (1966).

34 J. J. Sakurai, Advanced Quantum Mechanics (Addison-Wesley, Redwood, 1984).

35 A. R. Goñi, A. Pinczuk, J. S. Weiner, J. M. Calleja, B. S. Dennis, L. N. Pfeiffer, and K. W. West, Phys. Rev. Lett. 67, 3298 (1991).

36 J. Rubio, J.M. Calleja, A. Pinczuk, B.S. Dennis, L.N. Pfeiffer, K.W. West, Solid State Commun. 125 149-153 (2003).

37 D.W. Wang, A.J. Millis, and S. Das Sarma, Phys. Rev. B 64, 193307 (2001).

38 I.E. Dzyaloshinsky and A.I. Larkin, Zh. eksp. teor. Fiz. 65, 411 (1973) (English translation: Soviet Phys. JETP 38, 202 (1974)). 


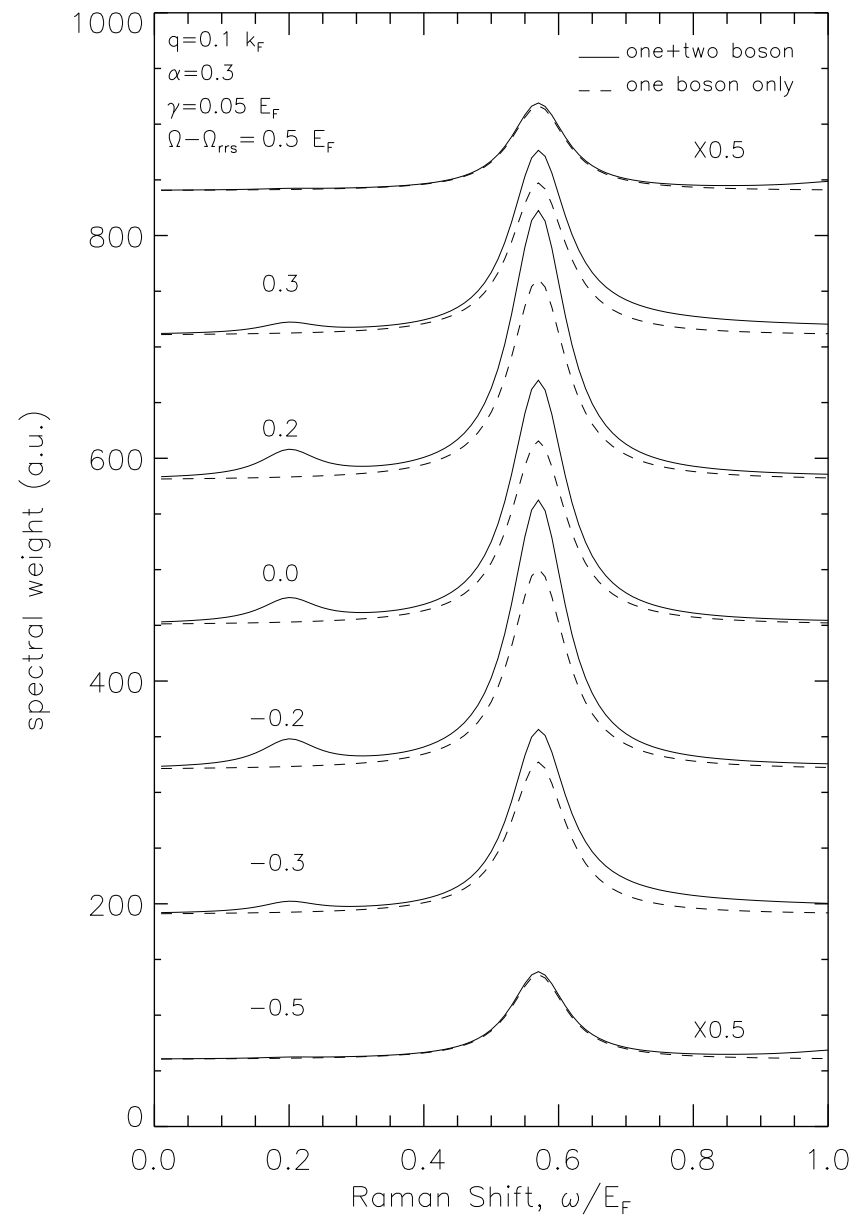

FIG. 2: Polarized RRS spectra calculated via the bosonic expansion method for various resonance conditions, $\tilde{\Omega}=\Omega-\Omega_{r r s}$. One- and two-boson contributions have been plotted separately in order to show their relative contributions (see text). Finite broadening factor $\gamma$ is introduced to express the delta function. Note that the overall spectral weights decreases dramatically off-resonance, as indicated by the individual scale factors on the right hand side of each plot. 


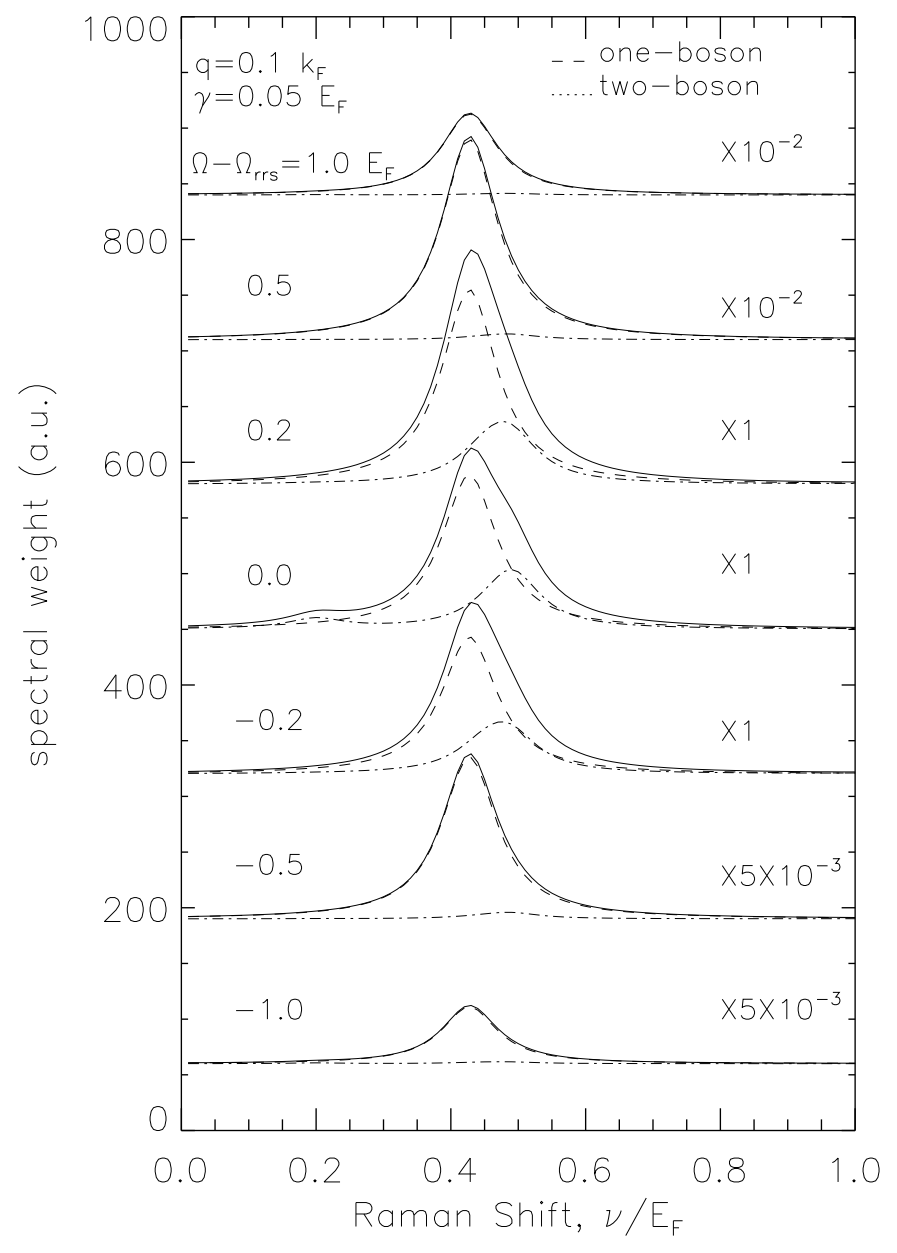

FIG. 3: Polarized RRS spectra calculated via bosonic expansion method for various resonance conditions in the LL model with long-ranged Coulomb interaction. One- and two-boson contributions have been plotted separately in order to show their relative contributions (see the text). Finite broadening factor $\gamma$ is introduced to plot the delta function contribution.

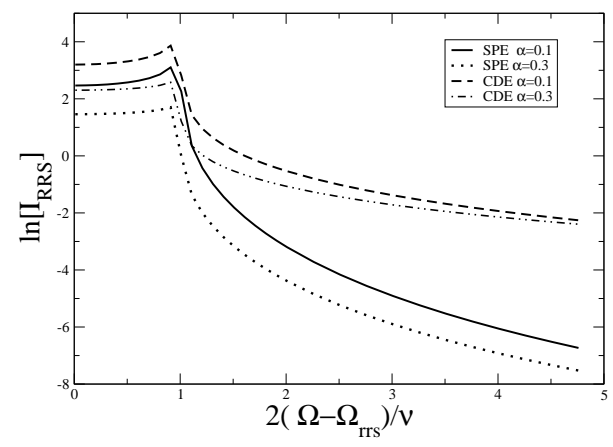

FIG. 4: Logarithm of polarized-channel Raman scattering cross section (normalized to appropriate mode velocity), $I_{R R S}$, plotted against incident laser frequency (normalized to mode excitation frequency $\nu=v_{\rho, \sigma} q$ and measured from the average of incident photon resonance energy and outgoing photon resonance energy with respect to the resonance energy, $\left.\Omega_{r r s}\right)$, for two different values of the Luttinger exponent $\alpha$. The plotted intensities are coefficients of delta functions describing coherent charge (CDE) and spin (SPE) final states and are computed via Luttinger liquid methods as described in the text 


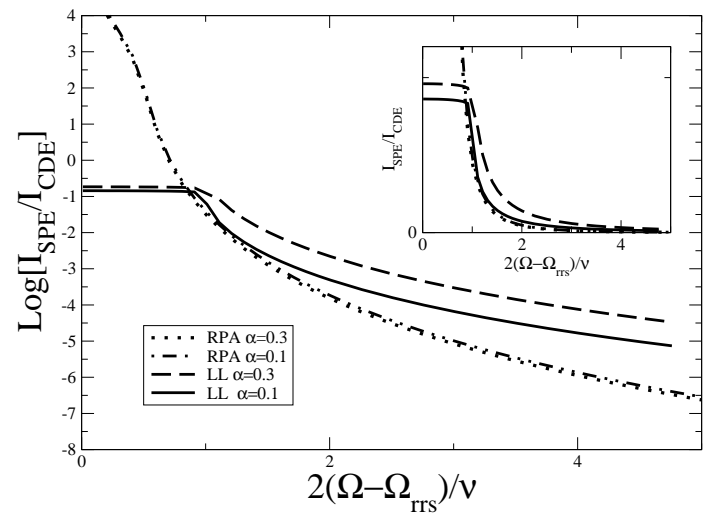

FIG. 5: Comparison of ratio of delta coefficients for CDE and SPE absorption for Luttinger (solid and long-dahsed lines) and RPA-Fermi liquid (dash-dot and short-dashed lines) model, normalized to appropriate mode frequencies and plotted against incident laser frequency (scale as in Fig. 4) Inset: same ratio, displayed in linear scale.

(1)

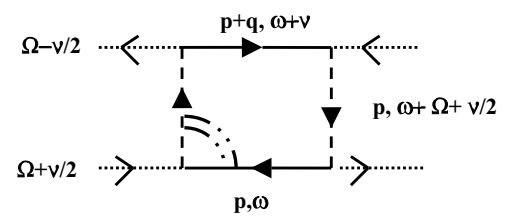

(2)

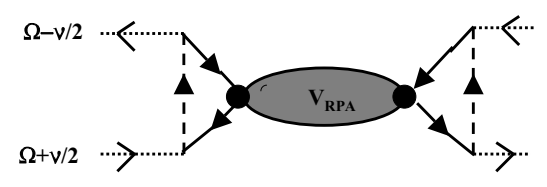

FIG. 6: Feynman diagrams considered in evaluation of 'RPA' approximation to unpolarized RRS cross section. Diagram 1 gives the dominant contribution to the SPE absorbtion. Excitonic interactions (such as those shown as dash-dot lines in the lower left corner of this diagram) are not considered in this paper. Diagram 2 gives the dominant contribution to the CDE absorbtion. 